\title{
Differential Sensitivity of Children and Adults to Chemical Toxicity
}

\author{
II. Risk and Regulation \\ Michael Dourson, ${ }^{*}$ Gail Charnley, $\dagger$ and Robert Scheuplein $\ddagger$ \\ *Toxicology Excellence for Risk Assessment, 1757 Chase Avenue, Cincinnati, Ohio 45223; †HealthRisk Strategies, 826 A Street SE, \\ Washington, DC 20003; and $\ddagger$ Keller and Heckman, 9 Meridian Road, Stafford, Virginia 22554
}

Received January 15, 2002

\begin{abstract}
Animals can be useful predictors of chemical hazards to humans. Growth and development are compressed into a shorter period in animals, which makes interpretation of animal testing inherently more difficult. However, similar events occur in both humans and laboratory animals and testing that covers the full period of animal development can reasonably be considered an appropriate surrogate for human development. Some have proposed an additional 10-fold factor for the extra protection of children when estimating safe exposures. Use of such an additional factor, as required by the Food Quality Protection Act (FQPA), is meant to address the same issues covered by the EPA's database uncertainty factor, $U_{D}$, and additional issues related to exposure uncertainty. Thus,

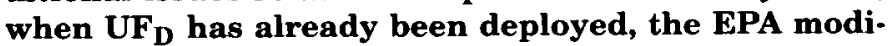
fies its use of the FQPA factor. Based on our analysis, we agree with the EPA. Drawing conclusions about the adequacy of $U F_{H}$, the uncertainty factor used to account for intrahuman variability, in terms of its ability to protect children on the basis of the modest data available is challenging. However, virtually all studies available suggest that a high percentage of the population, including children, is protected by using a 10 -fold uncertainty factor for human variability or by using a 3.16-fold factor each for toxicokinetic and toxicodynamic variability. Based on specific comparisons for newborns, infants, children, adults, and those with severe disease, the population protected is between 60 and $100 \%$, with the studies in larger populations that include sensitive individuals suggesting that the value is closer to $100 \%$. 2002 Elsevier Science (USA)
\end{abstract}

\section{INTRODUCTION}

One of the goals of chemical regulation is to limit potential risks of chemical toxicity. To do so effectively, regulators aim to limit chemical exposures. Determining the extent to which exposures must be limited in order to minimize risks to children and adults requires knowledge of the qualitative and quantitative charac- teristics of chemical hazards. The qualitative nature of hazards can be characterized through appropriate toxicity testing using laboratory animals. Understanding age-related quantitative differences in sensitivity is more challenging.

To a large extent, the body of U.S. laws that seek to establish practices that will ensure safety-or at least mitigate risk-from chemical or other contaminant exposures provided the impetus for the development of methods to identify appropriate quantitative limits on chemical exposures. Most of the methods used today by regulatory agencies were developed in reaction to the calls by these laws to define limits on exposure that will "protect the public health with an adequate margin of safety" or lead to "a reasonable certainty of no harm." That is, in passing the laws, the U.S. Congress called on the regulatory agencies to develop means to assess risks from chemicals or other agents so as to define exposure levels that would achieve the stated qualitative goals of health protection (Rhomberg, 1997).

Limiting exposures to chemical toxicants that achieve the public health goals set out by Congress often begins with the identification of "safe" or "virtually safe" doses. For example, the U.S. Food and Drug Administration (FDA) uses acceptable daily intakes to limit chemical exposures through food, the U.S. Occupational Safety and Health Administration uses permissible exposure levels to limit chemical exposures in the workplace, and the U.S. Environmental Protection Agency (EPA) uses reference doses (RfDs) and reference concentrations $(\mathrm{RfCs})^{1}$ to guide efforts to limit oral and inhalation exposures to chemicals, respectively.

The process of identifying safe doses begins with the identification of no-observed-adverse-effect levels

\footnotetext{
1 Please note that the majority of data that we discuss are for the oral route of exposure and, therefore, any conclusions that we draw should probably be restricted to this route. However, we anticipate that if sufficient inhalation data were available, similar conclusions might be drawn, especially if the critical effect is not limited to a portal of entry. We encourage additional analyses of inhalation data to test this supposition.
} 
TABLE 1

Description of Typical Uncertainty and Modifying Factors in Deriving Reference Doses (Rf Ds) or Reference Concentrations (RfCs) ${ }^{a}$

Standard Uncertainty Factors (UFs)

H (interhuman)

A (laboratory animal to human)

S (subchronic to chronic)

, (LOAEL to NOAEL:

D (incomplete data base to complete)

Modifying factor (MF)
General guidelines $b$

Generally use a 10-fold factor when extrapolating from valid experimental results from studies using prolonged exposure to average healthy humans. This factor is intended to account for the variation in sensitivity among the members of the human population.

For RfDs, generally use a 10-fold factor when extrapolating from valid results of long-term studies on experimental animals when results of studies of human exposure are not available or are inadequate. For $\mathrm{RFC}_{3}$, this factor is reduced to 3-fold when a NOAEL (HEC) is used as the basis of the estimate. In either case this factor is intended to account for the uncertainty in extrapolating animal data to humans.

Generally use a 10-fold factor when extrapolating from less than chronic results on experimental animals or humans. This factor is intended to account for the uncertainty in extrapolating from less than chronic NOAELs to chronic NOAELs.

Generally use a 10-fold factor when deriving an RfD or RfC from a LOAEL, instead of a NOAEL. This factor is intended to account for the uncertainty in extrapolating from LOAELs to NOAELs.

Generally use a 10-fold factor when extrapolating from valid results in experimental animals when the data are "incomplete." This factor is intended to account for the inability of any single study to adequately address all possible adverse outcomes.

Use professional judgment to determine an additional uncertainty factor termed a modifying factor (MF) that is greater than zero and less than or equal to 10. The magnitude of the MF depends upon the professional assessment of scientific uncertainties of the study and database not explicitly treated above (for example, the number of animals tested). The default value for the MF is 1 .

\footnotetext{
Note The maximum uncertainty factor used with the minimum confidence database for an $\mathrm{RfD}$ is 10,000 ; for an $\mathrm{RfC}$ it is $\mathbf{3 0 0 0}$.

a Source. Adapted in part from Dourson and Stara (1983), Barnes and Dourson (1988), Jarabek et al. (1994, 1995), and Dourson (1994).

${ }^{b}$ Professional judgment is required to determine the appropriate value to use for any given UF. The values listed in this table are nominal values that are frequently used by the EPA.
}

(NOAELs) or of doses that elicited a specific rate of response (benchmark dose, BMD), usually through laboratory animal testing. Laboratory animals are useful surrogates for humans, being similar in many respects. Humans may be more or less sensitive than laboratory animals. Laboratory animals are normally inbred and their responses to chemicals tend to be relatively uniform and consistent. For the purposes of regulation, humans are generally assumed to be more sensitive than the most sensitive species evaluated. Furthermore, permissible chemical exposure levels for humans must be safe for a variety of ethnic and otherwise dissimilar groups with inherently variable responses to chemical agents. In addition, laboratory animals are healthy and receive good nutrition; the same cannot be said for all people. For these reasons and for other special reasons described below, regulatory agencies have traditionally used "safety" or "uncertainty" factors to determine allowable levels for human exposure. Those levels are typically 100 -fold or more below the doses that produce no adverse effect in the most sensitive laboratory animal studies.

The basic equation to determine a safe dose is

Safe dose $=$ critical effect level/uncertainty factor(s)

Brief descriptions of commonly used safety or uncer- tainty factors (UFg) are provided in Table 1. These factors are reductions in the dose, based on scientific judgments of available toxicity, toxicodynamic, and toxicokinetic data and inherent uncertainty, necessary to identify an exposure level that is considered unlikely to produce adverse effects. The six specific categories shown in Table 1 follow the approach of the EPA. Although not all health organizations apply these factors as discrete divisors, most experts consider uncertainties in the following areas:

- extrapolation from shorter term exposures to longer term or lifetime exposures;

- absence of a NOAEL ${ }^{2}$;

- absence of adequate studies relevant to characterizing hazards;

- intrahuman variability;

- interspecies variability.

Several good reviews of this area are available (e.g., Dourson et al., 1996; Kalberlah and Schneider, 1998).

\footnotetext{
${ }^{2}$ NOAEL: The highest exposure level at which there are no statistically or biologically significant increases in the frequency or severity of adverse effect between the exposed population and its appropriate control; some effects may be produced at this level, but they are not considered adverse, nor precursors to adverse effects (EPA, 2000).
} 
Concern about children's potentially greater sensitivity to chemical toxicity is an important issue only in the context of potentially greater risk. Children are at greater risk of chemical toxicity if their exposures are high enough to produce adverse effects, whether they are more or less sensitive than adults. The question then is, are current regulatory approaches to limiting chemical exposures sufficient to protect children from exposures that are toxic; or, are chemical exposures misregulated due to inadequate attention to children's sometimes greater sensitivity to particular chemicals, putting them at greater risk? This discussion generalizes the types of uncertainty listed above to answer the question of whether current regulatory approaches to determining safe chemical exposures are adequate to protect children. Specifically, it evaluates three questions:

- Is the uncertainty factor used to compensate for the absence of comprehensive toxicity testing adequate to protect children?

- Does the uncertainty factor used to account for the diverse sensitivity to toxicity among individuals protect children as well as adults?

- Are these two factors together adequate to protect children?

\section{DETEGTING AND GHARACTERIZING DEVELOPMENTAL HAZARDS}

Toxicity testing plays a critical role in the detection and characterization of developmental hazards. Toxicity screening protocols can help flag potential developmental toxicants that should be subjected to further testing if widespread human exposure is likely. Further testing involves comprehensive protocols that have been developed to cover different periods of animal maturation in ways that are comparable to human development. For example, Fig. 1 shows a series of toxicity tests that covers most of the animals' life stages.

\section{Interspecies Temporal Concordance}

Although infant and adult nonhuman animals differ in much the same way that human infants and adults differ, there are substantial interspecies differences among the young. For example, the newborn mouse or rat more nearly resembles the human fetus in the third trimester of gestation than the human infant at birth (NAS, 1983). However, the rates of maturation and growth of the mouse or rat after birth are much more rapid than those of the human infant. Maturity of a rat or mouse after weaning (68 weeks) does not appear to lag far behind the comparable time in the human infant (6-8 months). But, as indicated in Fig. 2, individual organ systems develop at different rates in different species. For example, as a percentage of mature weight, the human brain at 15-20 months of age is similar to the rat brain at 1317 days of age (a roughly 30:1 temporal ratio) (Himwich, 1973).

The age-weight curves in Fig. 2 do not usually indicate functional maturity; most organs in the human are not fully mature functionally before they reach their final size. But a child or an animal at birth is reasonably well prepared for the abrupt changes that occur at parturition and most functional systems, although immature, possess a significant portion of their adult capacity. The growth curves also demonstrate a similar overall pattern of development among humans, mice, and rats based on physiological time.

Because of the different rates of maturation of specific functional systems in humans and animals, it is difficult to conduct cross-species temporal extrapolations between developing humans and developing animals. On the other hand, as long as the full course of development is tested in laboratory animals, there is every reason to believe that the same developmental processes occur sooner or later in humans and that the converse is also true. For most chemicals known to cause developmental effects in human, at least one animal species has been found to demonstrate similar effects (Hemminki and Vineis, 1985: Kimmel et al., 1990; Stanton and Spear, 1990).

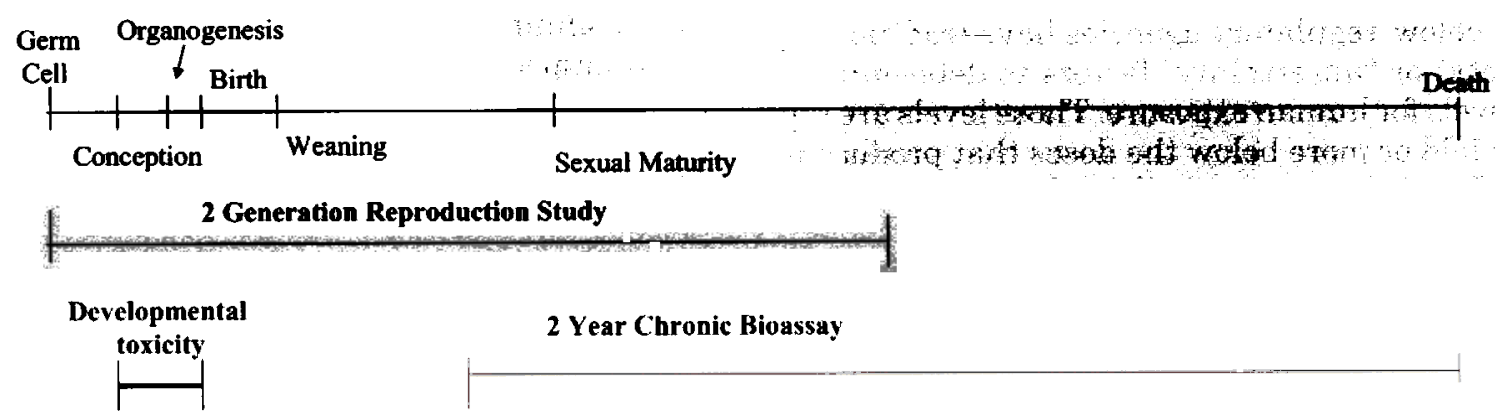

FIG. 1. Animal lifespan in relationship to the timeline of existing toxicity tests (time frames are not to scale). 


\section{A. Human}

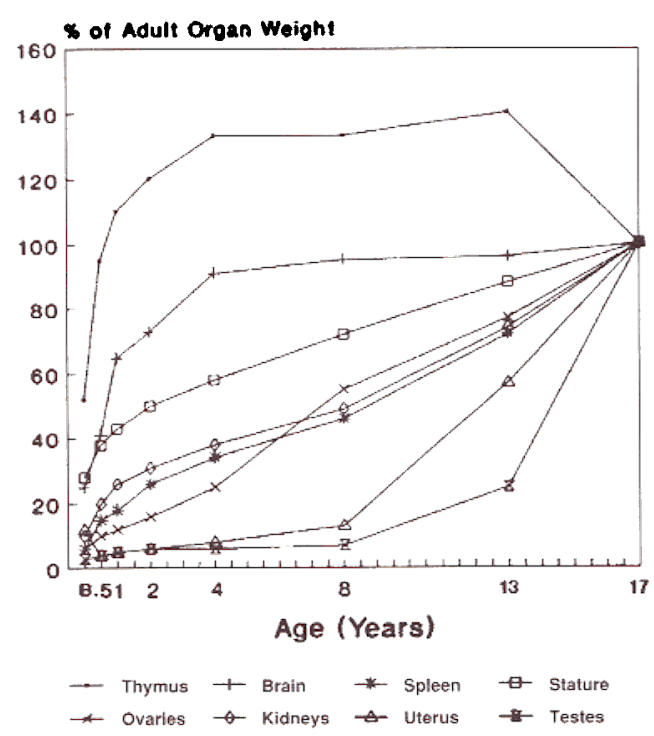

B. Rat

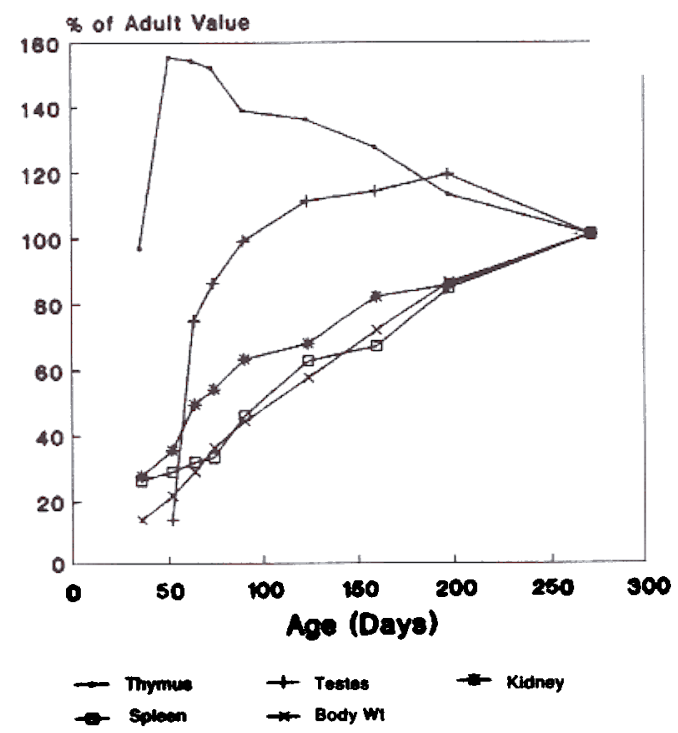

\section{Mouse}

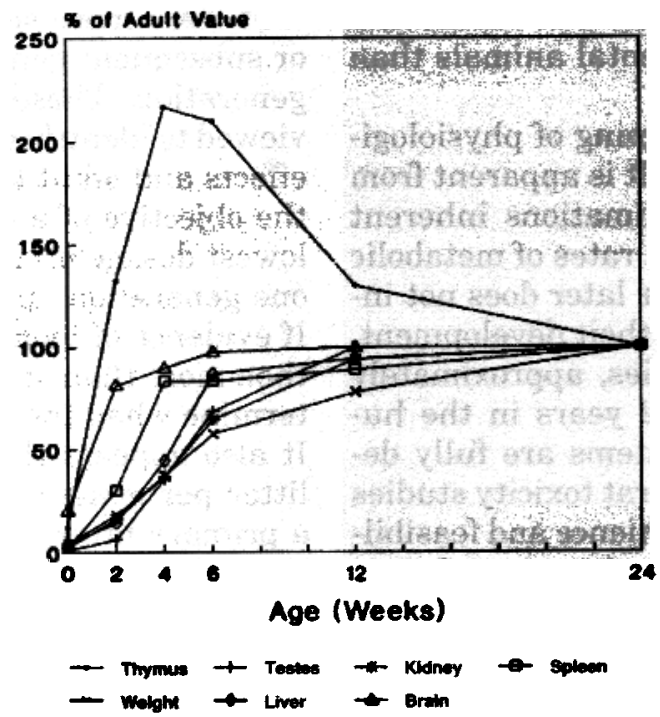

FIG. 2. Organ development and stature or body weight as a percentage of adult values by age. Source. Based on data from Altman and Dittmer, 1962.

\section{Developmental Toxicity Testing Protocols}

Developmental toxicity testing protocols have been developed by national regulatory agencies and by other, multinational groups. Most of those protocols are similar and are designed to expose test animals in all the ways that humans may be exposed: prior to and during pregnancy and during nursing and postnatal life. Despite the comprehensive nature of such protocols and observed interspecies concordance, concerns have arisen over their adequacy and sensitivity due to the difficulty in fully understanding the similarities and dis- parities of important developmental milestones of the test species when compared with humans.

The embryos of mammals, including humans, are susceptible to common external influences, including nutritional deficiencies, intrauterine infections, mechanical problems, and chemical agents. Because of the rapid changes occurring during development, the nature and sensitivity of the embro/fetus as a target for toxicity is also changing. The various developmental stages are relatively compressed in experimental animals. For example, the period of gestation is 21-22 days in the rat and 267 days in humans (Casarett and Doull, 2001). 
TABLE 2

A Rough Comparison of Physiological Maturation in Humans and Rats ${ }^{a}$

\begin{tabular}{|c|c|c|c|}
\hline Time period & Human & Rat & Comment \\
\hline & $\begin{array}{l}\text { Birth to } \\
1 \text { month }\end{array}$ & $\begin{array}{r}\text { Birth to } \\
1 \text { day }\end{array}$ & $\begin{array}{l}\text { Transition from intrauterine } \\
\text { environment to own } \\
\text { capacity }\end{array}$ \\
\hline Infancy & 1 month & $>1$ day & $\begin{array}{l}\text { Lower metabolic capacity of } \\
\text { many enzyme systems; } \\
\text { period of rapid } r\end{array}$ \\
\hline Childhood & 6 months & $>5$ days & $\begin{array}{l}\text { Activity of most metabolic } \\
\text { enzyme systems near, at, or } \\
\text { greater than adult levels }\end{array}$ \\
\hline Adolescence & 12 years & $>8$ weeks & $\begin{array}{l}\text { Transition to adult metabolic } \\
\text { activity; typical start time } \\
\text { for subchronic and chronic } \\
\text { studies in rats }\end{array}$ \\
\hline Adult & 18 years & $>6$ months & $\begin{array}{l}\text { Typical start time for clinical } \\
\text { testing in humans and } \\
\text { continued testing in rats }\end{array}$ \\
\hline
\end{tabular}

${ }^{a}$ Scheuplein et al. (2002) in part.

Accordingly, the critical windows of vulnerability during the various stages of development occur earlier and are more closely spaced in experimental animals than in humans.

Table 2 compares a very rough timing of physiological maturation in rats and humans. It is apparent from this chart that, despite the approximations inherent in assuming comparable maturation rates of metabolic systems, exposing rats at 6 weeks or later does not include the most vulnerable periods of their development. The typical start time for rat studies, approximately 8 weeks, corresponds roughly to 12 years in the human child, well after metabolic systems are fully developed. The 8-week start period for rat toxicity studies is largely a matter of practical convenience and feasibility. Rats and mice much younger than about 8 weeks are more difficult to handle and to subject to test protocols. The result is that the most vulnerable period in the child's development is not directly evaluated by routine chronic and subchronic tests in animals. Nonetheless, chronic and subchronic tests have value in assessing potential risks to children by, for example, identifying target sites for toxicity and providing dose-response information that may be useful for human safety assessment, irrespective of life stage.

To compensate for the period of development not covered by routine chronic and subchronic toxicity testing, reproductive toxicity studies that expose the developing animal in utero have been used since the early 1940s. In these multigenerational studies, the parent animals $\left(P_{0}\right)$ are exposed through the period from weaning to mating, the $P_{0}$ mothers are exposed through pregnancy and weaning, and their progeny $\left(F_{1}\right)$ are exposed until they mate. For the $F_{1}$ offspring this protocol includes in utero exposure, exposure through mother's milk and, after weaning, oral exposure through the diet. The new $\left(F_{1}\right)$ mothers are also exposed through their pregnancy and weaning. For the offspring of $F_{1}$ parents, referred to as $F_{2}$, this protocol also includes in utero exposure and exposure through their mother's milk until weaning. Because of the immaturity of metabolic and physiologic systems, the first 6 months of the human infant period, corresponding approximately to $1-3$ days in the rat, is the most critical from the viewpoint of potential developmental effects. Although the fetal stage is often considered the most vulnerable, the fetus appears to be adequately evaluated by standard developmental toxicity studies in two species and in two generations of the reproductive study. It is the immediate postnatal period that has garnered recent attention from toxicologists, because it is difficult to conduct extensive toxicity tests on newborn animals.

Christian (1986) reviewed 817 reproductive and developmental studies against rigorous criteria to evaluate the usefulness of results from multiple generations when compared to a single generation. Seventy-three studies passed the criteria, of which 38 reported positive results. Twenty of those positive studies showed effects that were more severe or first detected in the second or subsequent generations when compared to the first generation. These 20 studies were then critically reviewed to identify adult and litter primary reproductive effects and adult toxicity. The author concluded that if the objective of a reproductive study is to identify the lowest dosage producing a primary reproductive effect, one generation appears to be sufficient for evaluation. If evidence of bioaccumulation of the agent is evident, then more than one generation may be necessary to determine when the steady state of the agent is attained. It also appears that the evaluation of more than one litter per generation is not necessary to demonstrate a primary litter reproductive effect. Since the publication of this work, testing guidelines have been updated because of questions regarding the adequacy of older studies. It would be of interest to repeat this work on the basis of newer studies.

The United States, Japan, and the United Kingdom use a three-segment protocol for the evaluation of new pharmaceutical agents. Segment $I$ is designed to evaluate fertility and general reproduction and to assess potential developmental effects in the offspring. Segment $I$ is conducted using one species (usually the rat) and involves the treatment of males and females prior to conception and the continued treatment of females throughout gestation and lactation. Segment II is a teratology or developmental study, usually conducted in two species (usually mice, rats, or rabbits). Segment III is designed to evaluate peri- and postnatal toxicity in exposed dams and their offspring in one species (usually the rat). The highest dose used in all three segments generally is required to induce some form of minimal toxicity in the animal to ensure that an 
adequate dose range is covered. FDA protocols for food additives include tests designed to detect effects on gonad function, estrous cycles, mating behavior, conception, parturition, pregnancy outcome, lactation, and postnatal growth and viability for up to three generations. Various agencies augment standard protocols by requiring behavioral assessments in Segment II and III studies.

The developmental toxicity study protocols used by the EPA and OECD are almost identical to the FDA Segment II teratology study. Those protocols are designed to evaluate the effects on soft tissues and skeletal development from exposures in utero during the periods of histogenesis and organogenesis.

The EPA (1991) published developmental neurotoxicity (DNT) testing guidelines (Supplement 10) for evaluating potential functional and morphological impacts of toxicants on the nervous system that may arise in offspring from exposure of the mother during pregnancy and lactation. Those guidelines have been recently updated by the EPA's Office of Prevention, Pesticides and Toxic Substances (U.S. EPA, 1998). The dosing period includes exposure in utero (starting gestation day 6) and during the postnatal period (through postnatal day 10). Animals are observed for 60 days for effects on developmental, behavioral, and neurological endpoints. Behavioral endpoints include learning and memory tests. The neurological observations include tests of motor activity and auditory startle; brain weights and neuropathological evaluations, including brain neuropathology, are recorded at the end of the study. Modifications of this protocol have been suggested, but whether this protocol or its modifications is acceptable as a useful adjunct to the traditional protocols needs further discussion within the scientific community. The fundamental problem with the new DNT testing requirements is that there are no published data demonstrating that such testing will reveal any neurological damage not also obtainable using a well-conducted two-generation reproductive study. The EPA does not recommend DNT testing for all substances, but only on a case-by-case basis depending on the toxicological information available for the chemical or class of chemical of interest. The EPA's Office of Toxic Substances has developed criteria for when DNT testing will be required (Francis et al., 1990; U.S. EPA, 1998).

\section{Data Needs for "Safe" Dose Assessment}

The specific number and types of toxicity tests used for safety assessment vary considerably across regulatory programs. The registration requirements of different countries for substances developed for specific biological activity, such as food use pesticides, are most stringent and can include many distinct mammalian toxicity tests. Similar requirements apply to pharmaceutical agents. Other assessment approaches, such as the OECD (1997) Screening Information Data Set (SIDS) process, follow a tiered approach, in which a base set of toxicity studies is evaluated initially and, depending upon the results, the need for additional studies is determined. Similarly, the EPA's approach for evaluating "inert" ingredients in pesticide formulations consists of a base set of tiered toxicity studies and guidelines for interpretation of results that lead to the triggering of more extensive toxicity studies.

Tiered approaches are typically applied to substances that are not specifically designed to be biologically active. Such chemicals differ in physical/chemical characteristics and in many other ways from pesticides and pharmaceuticals. Further, production processes and use patterns influence or limit intentional human exposures. An approach that begins with a limited core set of toxicity tests, such as the OECD (1997) SIDS, provides an efficient means of evaluating substances within the framework of a screening-level safety assessment and leads to setting additional testing priorities based on both toxicity concerns and exposure potential.

In the determination of safe doses from such studies, few investigators have discussed or agreed upon what comprises the necessary amount of appropriate data (see, for example, early work of Clegg, 1978). However, the EPA has used an uncertainty factor (based in part on earlier work of FDA) to estimate safe exposure levels in the absence of adequate data from multiple toxicity studies (Barnes and Dourson, 1988). This factor is now referred to as $U_{D}$ (Dourson, 1994). The EPA considers this factor necessary because of the inability of any one study to adequately test different species or different life stages of the same species. The EPA has often found that the receipt of missing studies yields a different critical effect and a lower NOAEL. The EPA's use of UF $_{D}$ is based on its assumption that the critical effect can be discovered in a reasonably small selection of toxicity studies. In the context of setting safe exposure limits that protect children, evaluating the adequacy of and need for $U_{D}$ has become important because of concerns that incomplete toxicity testing will fail to identify effects relevant to children's health.

Initial attempts to understand how different toxicity studies identified the critical effect for safe exposure limits naturally focused on the frequency of different critical effects in the determination of such limits (see, for example, Fig. 3). Such evaluations included systemic toxicity in laboratory animals through acute, short-term, subchronic, and chronic studies; specialized testing, such as evaluations of developmental toxicity, reproductive toxicity, immunotoxicity, and neurotoxicity; and toxicokinetic and toxicodynamic evaluations. If available, all of these studies are used to characterize a chemical's spectrum of potential human toxicity by identifying target organs and the dose ranges 


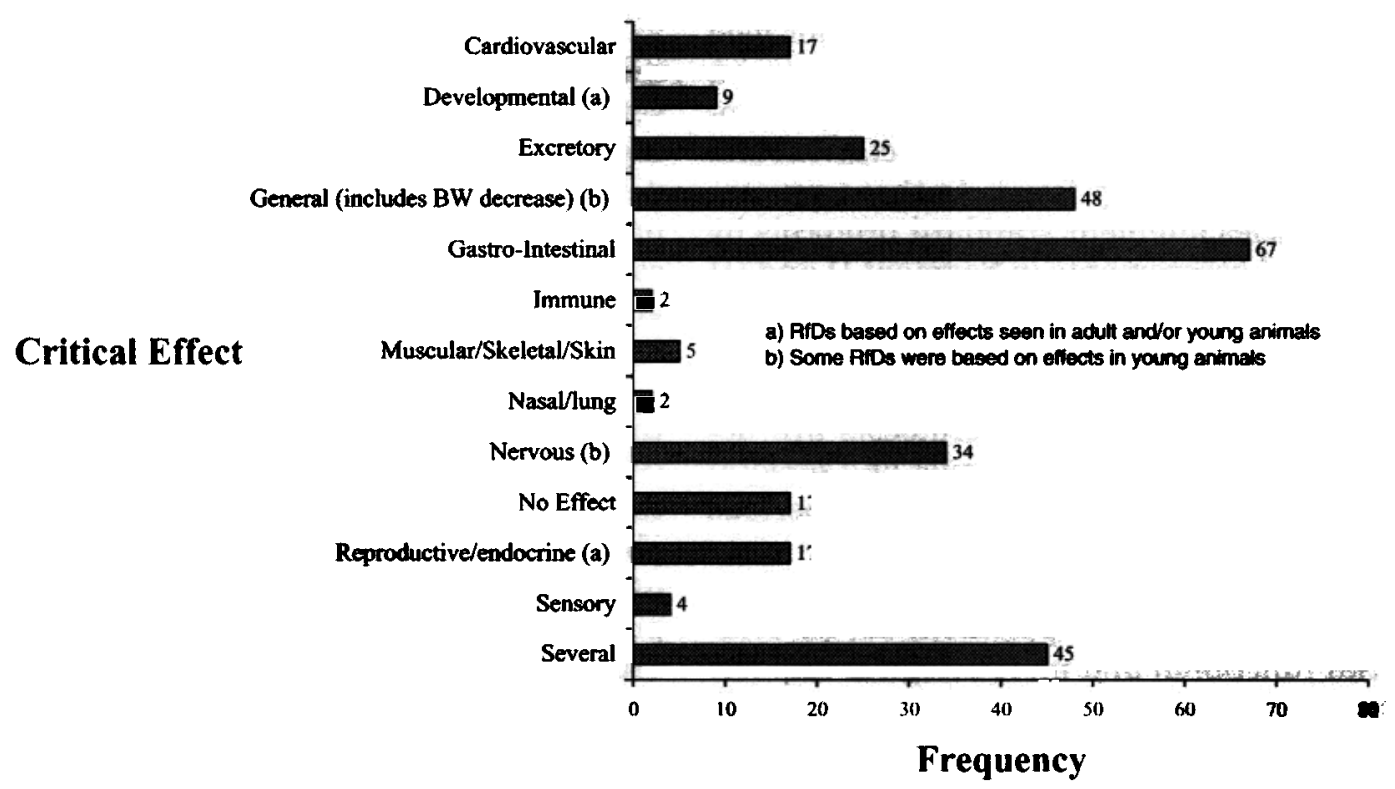

FIG. 3. Distribution of critical effects for $292 \mathrm{Rf}$ D's on the EPA's IRIS as of 7/1/95.

associated with adverse effects in laboratory animals of different life stages. ${ }^{3}$

Unfortunately, a problem with these initial evaluations is quickly evident. Quite simply, the databases for many chemicals lack a sufficient number of studies that evaluate different endpoints and life stages. Thus, the results in Fig. 3, which illustrate that $\sim 9 \%$ of all EPA RfDs are based on reproductive or developmental toxicity studies, do not give much assurance that this percentage represents an accurate estimate of the number of times these effects might serve as the basis of exposure limits if complete chemical-specific databases were more widely available.

The EPA conducted further work on the impact of missing data in developing RfDs, including data for different life stages, and this research directly relates to evaluations of the usefulness of $\mathrm{UF}_{\mathrm{D}}$ (Dourson et al., 1992). For example, data for 69 pesticides were analyzed and frequency histograms of NOAEL ratios were developed for chronic dog, mouse, and rat toxicity studies and for rat reproductive and developmental toxicity studies (see Fig. 4). These pesticides were selected

\footnotetext{
${ }^{3}$ In vitro data can be used to elucidate potential mechanisms of biological activity, to evaluate the relevance to humans of the endpoint observed in laboratory animals, to improve extrapolation from laboratory animals to humans, and to characterize intrahuman variability. Assessment of laboratory animal data should include an evaluation of the reliability of the experimental design and toxicological interpretation of the results. Moreover, once a critical effect and likely mode of action have been identified, results from the various studies should be examined collectively to determine whether a causal relationship is likely to exist between a chemical exposure and the hypothetical human effect. Species-specific differences in sensitivity to a chemical due to differing metabolism, physiology, or anatomy, also should be considered.
}

because of the availability of many different toxicity studies on both adult and young animals. On average, chronic rat and dog studies, generally conducted on young adult to older animals, yielded similar NOAELs. Reproductive and developmental toxicity studies, conducted on both adult and young animals, were less likely to produce the lowest NOAELs when compared to the chronic rat and dog studies. Chronic mouse studies, generally conducted on young adult to adult animals, were least likely to yield the lowest NOAEL when compared to the chronic rat and dog studies and thus only occasionally resulted in the determination of a critical effect. The authors concluded that several bioassays are needed in order to develop a high confidence estimate for an RfD and, if one or more bioassays is missing (which is often the case when developing RfDs and other safe doses), then a factor such as $\mathrm{UF}_{\mathrm{D}}$ could be supported quantitatively. Specifically, when chronic rat and dog studies are available but rat reproductive and rat developmental toxicity studies are missing, a $U F_{D}$ of 3 applied to the lower of the chronic rat or dog NOAEL accounts for $\sim 92 \%$ of the possible occurrences of lower NOAELs being identified by the missing bioassays that include younger animals. $\mathrm{AUF}_{\mathrm{D}}$ of 10 accounts for $98 \%$ of such occurrences. ${ }^{4}$ Therefore, the routine use of $U F_{D}$

\footnotetext{
4 The specific comparison made is found in Dourson et al. (1992), Table 6, line 18. The values of 0.08 at $10^{0.5}$ and 0.02 at $10^{1.0}$ are the probabilities that either the rat reproductive or rat developmental toxicity study NOAELs are lower than the corresponding NOAELs for either the chronic dog or the rat bioassays. Thus, the chronic bioassay NOAELs, when divided by an uncertainty factor of $3\left(10^{0.5}\right)$ or $10\left(10^{1.0}\right)$, protect against either 92 or $98 \%$ of the potentially lower NOAELs that could be identified by bioassays that include younger animals, respectively.
} 


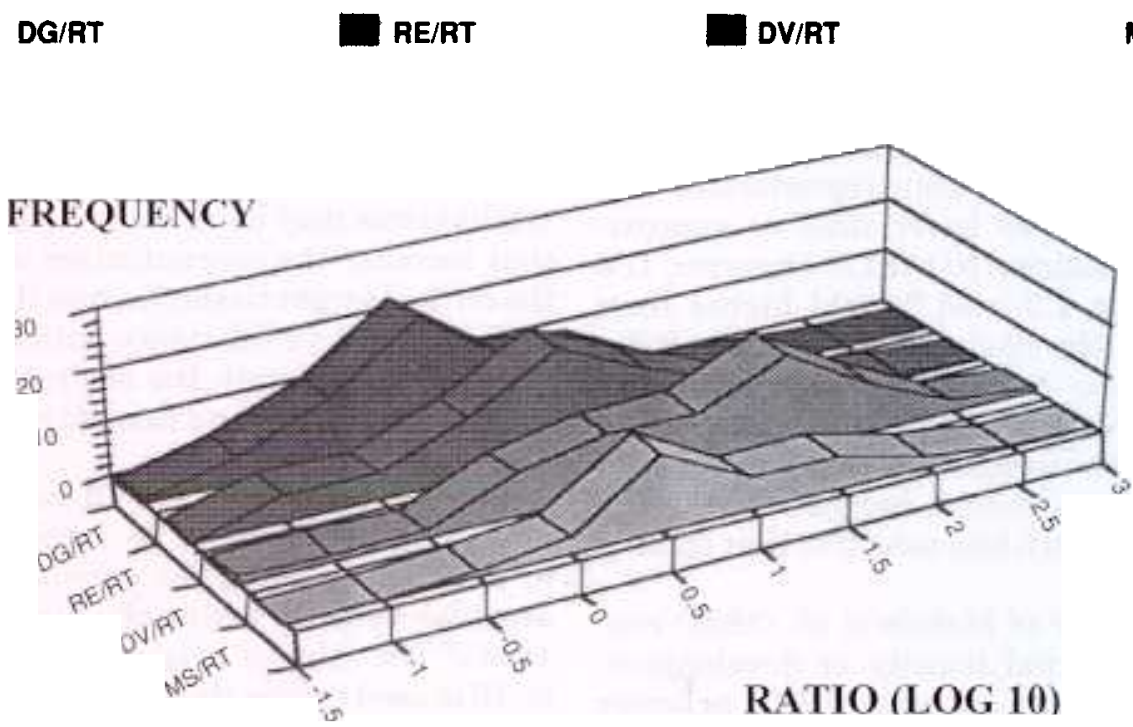

FIG. 4. The frequency of the $\log _{10}$ NOAEL ratios compared: chronic dog to chronic rat (DG/RT); reproductive rat to chronic rat (RE/RT); developmental toxicity rat to chronic rat (DV/RT); and chronic mouse to chronic rat (MS/RT). Zero value indicates equivalent NOAELs.

by the EPA to compensate for the lack of certain bioassays already addresses, in large part, the uncertainty associated with the absence of specific studies, including studies that test younger animals.

Baird et al. (1996) presented two approaches for estimating the quantitative value of $\mathrm{UF}_{\mathrm{D}}$ using a subset of studies on pesticides identified by Dourson et al. (1992), discussed above. One method, based on regression analysis, provided a point estimate of $\mathrm{UF}_{\mathrm{D}}$. The other method, based on nonparametric analysis, intended to provide a distributional estimate of $U F_{D}$. In both cases, the choice of $U_{D}$ depended on the definition of a complete database (see the EPA's described below), the number of missing bioassays, and the specific bioassay missing.

Based in part on the analysis of Dourson et al. (1992) of pesticides and published criteria for causal significance of Hill (1965), the EPA routinely uses $U F_{D}$ to determine $\mathrm{RfDs}$ in cases where certain bioassays are missing, including when studies are missing that test younger animals. This use allows the EPA to confidently develop RfDs for many compounds without the full complement of toxicity tests. The EPA generally considers a "complete" database ${ }^{5}$ - that is, complete for

\footnotetext{
${ }^{5}$ Generally, the presence of a complete database indicates that the acquisition of additional toxicity data is unlikely to result in a change to the RfD or RfC. Scientists at the EPA typically consider such RfDs and $\mathrm{RfCs}$ to be "high confidence," reflecting the likely stability of the value to additional data. The EPA considers a single, well-conducted, subchronic mammalian bioassay by the appropriate route as a minimum database for estimating a RfD or RfC. However, for such a limited database, the likelihood that additional toxicity data may change the value of the $\mathrm{RfD}$ or $\mathrm{RfC}$ is higher, and the associated confidence in them is lower. Due to the conservatism inherent in the uncertainty factor approach, the acquisition of additional
}

the purpose of estimating RfDs or RfCs for noncancer health effects with "high" confidence and no use of the database uncertainty factor-to comprise the following:

- two adequate ${ }^{6}$ mammalian chronic toxicity studies by the appropriate route of exposure in different species;

- one adequate mammalian multigeneration reproductive toxicity study by the appropriate route of exposure;

- two adequate mammalian developmental toxicity studies by the appropriate route of exposure in different species.

This series of tests is considered complete because most of the animals' life stages will have been investigated (see Fig. 1). The judgment of a complete database is somewhat chemical-specific, however; the observation of certain types of toxicity (e.g., neurotoxicity) in short-term tests may suggest the need for specialized tests not included in the general definition of a complete database.

For example, Makris et al. (1998) investigated the usefulness of developmental neurotoxicity (DN) tests

data often results in higher $\mathrm{RfDs}$ and $\mathrm{RfCs}$ (i.e., results in the conclusion that higher exposures are safe). For more details please U.S. EPA (1994) or Dourson (1994). Examples of confidence statements for RfDs and RfCs can be found in the EPA's online IRIS database (www.epa.gov/iris).

${ }^{6}$ As determined by professional judgment. Typically, studies should have been adequately conducted and published in refereed journals or be unpublished reports that adhered to Good Laboratory Practice guidelines and have undergone final QA/QC (U.S. EPA, 1994). The EPA and others have published guidelines in this area. For example, see U.S. EPA (1998) and FDA (1993). 
as part of this database by comparing DN NOAELs to NOAELs derived from other types of toxicity tests. They found that for nine of the pesticides investigated, eight DN NOAELs were lower than developmental toxicity NOAELs, six were lower than reproductive toxicity NOAELs, and six were lower than or approximately equal to neurotoxicity NOAELs. However, DN NOAELs were between 1.3- and 93-fold higher than the NOAELs used as the basis of the lifetime RfDs for seven of these same pesticides. For the remaining two pesticides, DN NOAELs were 70 and $90 \%$ of the chronic NOAELs. The mean difference between DN and chronic NOAELs was 25-fold, suggesting that DN NOAELs are generally much less sensitive that chronic NOAELs.

Moreover, a peer review of Makris et al. (1998) concluded that either maternal toxicity or developmental toxicity generally occurs at comparable or lower dose levels than developmental neurotoxicity (SAP, 1999). The peer review showed that for 10 of the 12 substances evaluated, either the maternal toxicity NOAEL or the developmental toxicity NOAEL was the same as or less than the DN NOAEL. In only 1 case was the DN NOAEL less than either the maternal toxicity NOAEL or the developmental toxicity NOAEL, and in this case the effect reported for the DN NOAEL was questioned by the peer review. With respect to the applicability and sensitivity of the DN study, the majority of the peer review panel strongly indicated that the DN study was not more sensitive than either the developmental study or the reproductive study.

Thus, while the DN study may (or may not) be more sensitive in some cases than other specialized studies, its overall contribution to the determination of a lifetime RfD is likely to be minimal, because it is not as sensitive as chronic bioassays. Its use in the development of higher acute or other less than lifetime RfDs is perhaps more likely, because in these situations, lifetime studies are seldom used.

Overall, an uncertainty factor of 3 or 10 commonly used by the EPA for varying degrees of database incompleteness seems appropriate and more than adequate when information suggests that developmental, reproductive, or developmental neurotoxicity may be the critical effect in the absence of specific information. ${ }^{7}$ This conclusion is based on a fair number of pesticides, but could be enhanced with reviews on other types of chemicals.

\footnotetext{
${ }^{7}$ In the case of specific information on these endpoints, the choice of NOAEL or LOAEL of the critical effect becomes more definitive. For example, when such endpoints are the critical effect, then the lifetime RfD is based on their NOAEL, even though the study is of shorter duration.
}

\section{VARIABILITY, UNCERTAINTY, AND DIFFERENTIAL SENSITIVITY}

Individual susceptibility depends on both toxicokinetic $^{8}$ and toxicodynamic mechanisms, and these mechanisms may be classified into three types: factors that increase the concentration of active substance at the critical target tissue; factors that augment the reaction of the active substance with the target tissue; and factors that promote the sequence of events between the initial reaction and final adverse effect (Grandjean, 1992).

An uncertainty factor of 10 , commonly referred to as $\mathrm{UF}_{\mathrm{H}}$, is generally used to account for the variability in response between the population mean and highly sensitive subjects within the human population (IPCS, 1994).$^{9}$ The value of 10 is used for $\mathrm{UF}_{\mathrm{H}}$ as a default; that is, 10 is used unless there are data indicating that a different value is more appropriate. Use of $\mathrm{UF}_{\mathrm{H}}$ assumes that there is variability in response to chemical toxicity from one human to the next and that this variability may not have been detected in the epidemiology study, usually due to factors such as small sample size. Use of this factor may also assume that groups of humans exist, such as children, the elderly, or those with genetic polymorphisms that predispose them to unique sensitivity when compared with the average population (e.g., a bimodal distribution of sensitivity; see below for further discussion). A recent review of this default factor indicates that it is relatively robust, with greater than 99\% of the population, including sensitive subgroups, being protected (Burin and Saunders, 1999).

\footnotetext{
${ }^{8}$ For the purposes of this text, we define toxicokinetics as the chemical's absorption, metabolism (excluding target tissue metabolism), distribution, and elimination. Others have defined toxicokinetics as above but without the exclusion of target tissue metabolism. We use our definition because of expediency. Quite simply, the majority of toxicokinetic data we analyze are measurements of AUC or clearance (see comparisons shown in Table 4), and these parameters appropriately represent the variability in kinetics that exclude target tissue metabolism. Furthermore, most of the studies looking at kinetic variability are looking at the parent compound. For pharmaceuticals for which the active agent is a metabolite, the active metabolite is usually (although not always) generated in a different tissue than the target tissue, and our definition of kinetics will properly note expected variability. In contrast, toxicity for environmental chemicals may more often result from generation of the active metabolite in the target tissue. Variability in this target tissue metabolism would not be included in our definition of toxicokinetics, and therefore our conclusions of toxicokinetic variability will be lacking. These are complex issues and will require additional research and discussion to fully sort out.

${ }^{9}$ While $U_{H}$ seeks to provide protection for sensitive members of the population, IPCS (1994) specifically states that "idiosyncratic hypersusceptibility (excessive reaction following exposure to a given dose of a substance compared with the large majority of those expased to the same dose) in a few individuals would not be the basis for the derivation of the TI [Tolerable Intake, which is symonymous with an RfD] ..." This caveat is used by other groups as well.
} 

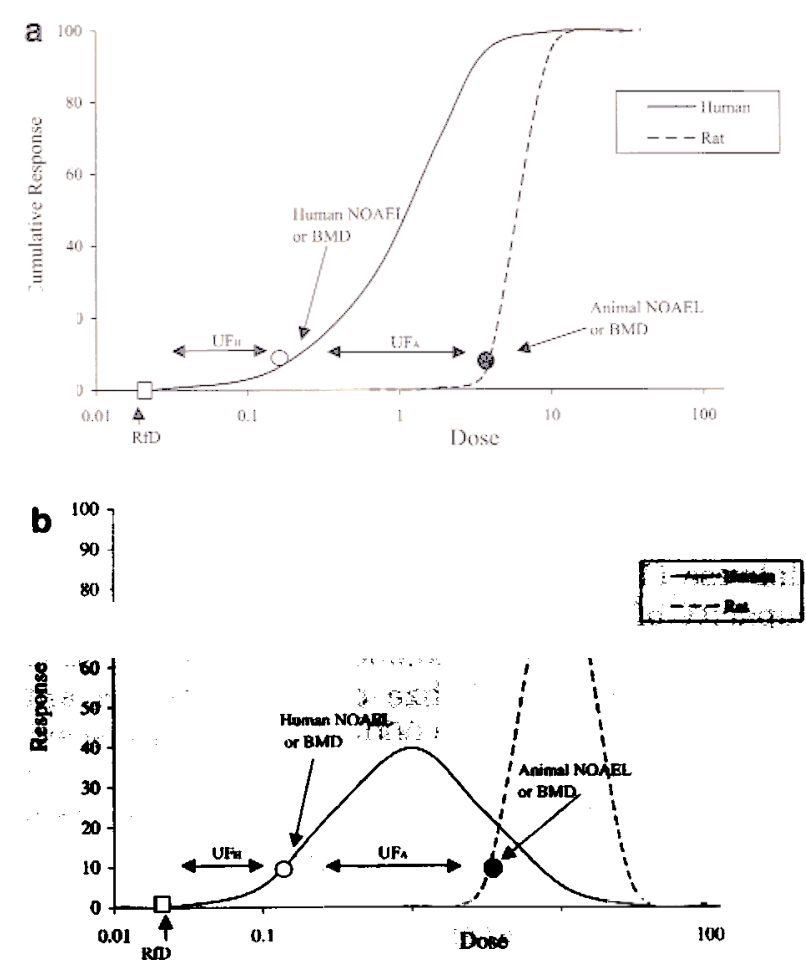

FIG. 5. (a) Cumulative response as a function of dose for humans and rats. Data are hypothetical, but approximate real situations. (b) Response as a function of dose for humans and rats. Hypothetical data are the same as in (a).

\section{Interpreting $U F_{H}$}

Significant misunderstanding about what $\mathrm{UF}_{\mathrm{H}}$ represents is apparent from the literature. The use of $U F_{H}$ applied to the NOAEL or BMD is not expected to reflect the complete distribution of human sensitivities, as some investigators in this area have suggested, or even the population mean to the highly sensitive subjects within the human population as mentioned above. Rather, applying $\mathrm{UF}_{\mathrm{H}}$ to the projected NOAEL or BMD reflects the range of sensitivities expected between the lower range of a normal distribution in the overall population and the sensitive subgroup (Dourson et al., 1996). ${ }^{10}$

$\mathrm{UF}_{\mathrm{H}}$ is commonly applied to the NOAEL or BMD estimated for humans. Human NOAELs or BMDs are usually projected from those observed in laboratory animals by dividing by another uncertainty factor, $\mathrm{UF}_{\mathrm{A}}$ (not discussed here), which is meant to account for differences in sensitivity between species (see Fig. 5a). This projected NOAEL or BMD for humans is expected to reflect the rate of response in the lower range of a

\footnotetext{
${ }^{10}$ Price et al. (1999) have written a very nice text that explains this issue using both a finite sample size model (which we represent in Fig. 5) and a sensitive population model (which we represent in Fig. 6).
}

normal distribution of human responses, because this is what the NOAEL or BMD reflects in the animal study (see Fig. 5b). In some cases the NOAEL and BMD can be measured or estimated from human studies. If so, some assurance is needed that the NOAEL and BMD are not derived from a subpopulation of resistant individuals. In that case, the NOAEL and BMD might not reflect the rate of response in the lower range of a normal distribution of human responses, by definition.

Figure 6a shows a trimodal distribution composed of sensitive, average, and resistant humans. ${ }^{11}$ When interpreted properly and starting from a NOAEL or BMD of the average group of humans, $\mathrm{UF}_{H}$ accounts for overall variability in the human population of much greater than 10-fold, perhaps between 100 - and 1000 -fold or more (see also Fig. 6b; variability spans approximately 3 orders of magnitude). Such appropriate interpretation also allows modification of $\mathrm{UF}_{H}$ when NOAELs are available for a known sensitive or resistant human subgroup or if human toxicokinetics or toxicodynamics are known with some certainty. In such cases, $\mathrm{UF}_{\mathrm{H}}$ should be adjusted (either increased or decreased) or replaced accordingly.

As an example of how one agency approaches the use of $\mathrm{UF}_{\mathrm{H}}$, Table 3 shows a comparison of $24 \mathrm{RfDs}$ based on human data as found on IRIS (U.S. EPA, 2001). Of these 24 values, 4 of them are based on a critical effect found in children, who are known to be the sensitive subgroup (fluorine, methyl mercury, nitrate, and nitrite). Five additional values are based on large population studies, which may have included children, but are also judged to include at least some sensitive individuals (arsenic, benzoic acid, cadmium, manganese, and selenium). In all but 2 of these 9 cases, $\mathrm{UF}_{\mathrm{H}}$ was reduced from its 10-fold default value to either 3 (arsenic and selenium) or even 1 (benzoic acid, fluorine, manganese, nitrate, and nitrite). Reductions of $\mathrm{UF}_{\mathrm{H}}$ are judged appropriate when sufficient data are available to suggest that there are unlikely to be any sensitive subgroups or where the RfD is based on a NOAEL or BMD from a sensitive human subgroup.

\section{Adequacy of $U F_{H}$ for Adults}

A number of scientists have investigated whether $\mathrm{UF}_{\mathrm{H}}$ accounts quantitatively for the variability to chemical toxicity between the overall human population and its potentially more sensitive groups. Such studies are most useful in addressing the adequacy of $\mathrm{UF}_{\mathrm{H}}$. Other investigators have generated data that are less useful for evaluating the adequacy of $\mathrm{UF}_{\mathrm{H}}$, because the research was done for some other purpose. Both types

\footnotetext{
11 Note here that the NOAEL or BMD appears to be less than the $10 \%$ mark shown more clearly in Fig. 5b. However, the NOAEL in Fig. $6 a$ is the same as that in Fig. 5b. In the case of Fig. 6a, two populations must be added to obtain the $10 \%$ response, that of the sensitive and average humans.
} 

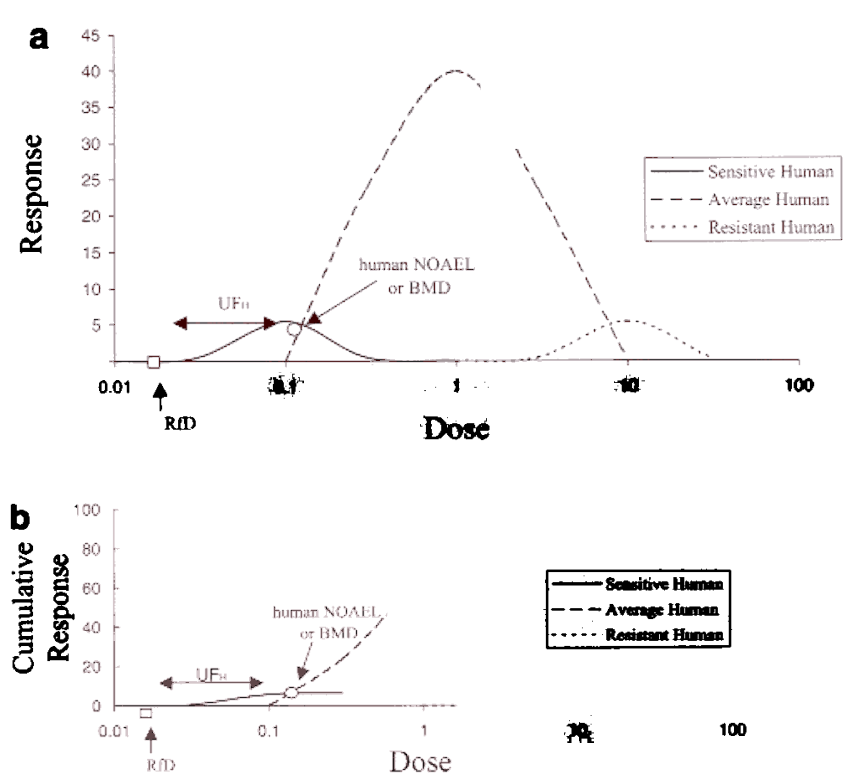

FIG. 6. (a) Response as a function of dose for humans of different sensitivities. Hypothetical data for humans are the same as in (b). (b) Cumulative response as a function of dose for humans of different sensitivities. Data are the same as in (a).

of studies are summarized in Table 4 and described below.

Dourson and Stara (1983) analyzed acute toxicity data for 490 chemicals from Weil (1972), finding that for about $92 \%$ of the chemicals, a 10-fold $U_{F}$ would yield a 3 -probit reduction from a median response (i.e., $99.9 \%$ of the population would be protected). Brown (2001) compared $\mathrm{ED}_{50}$ 's or $\mathrm{LD}_{50}$ 's for critically ill or injured patients or laboratory animals and found that a UF of 10 applied to the normal population's $E D_{50}$ or $\mathrm{LD}_{50}$ protected to the lower 5 th percentile of the compromised population $97 \%$ of the time. Hattis et al $(1999 \mathrm{a}, \mathrm{b})$ projected the incidence of effect that would be expected for a 10 -fold reduction of exposure from a $5 \%$ incidence level, assuming that population distributions of susceptibility are lognormal. This starting point is more like a NOAEL, so any resulting conclusions from this study have greater implications of applying a factor of 10 to a NOAEL, when compared to other studies. Results indicated that a 10 -fold reduction from the $5 \%$ level is associated with population effect incidences ranging from slightly less than 1 in 10,000 to a few per thousand. Brock (1991) evaluated the coefficient of variation in human susceptibility to plasma cholinesterase inhibition from organophosphate insecticide exposure among and within individuals. Mean variation among differing exposed and control groups of workers was between 10.9 and $14.0 \%$ while the range of individual variation within groups was as great as between 3.0 and $41.8 \%$. Men and women did not differ in this enzyme activity. Using a standardized measure of cholinesterase inhibi- tion, a 1.7-fold difference between the mean of the whole exposed population when divided by 2 standard deviations away from this mean can be determined, that is: $8.21 \mathrm{kU} / \mathrm{L} /(8.21 \mathrm{kU} / \mathrm{L}(2 \times 1.64))$. The comparable factor for the unexposed population is 1.5-fold.

Hattis et al. (1987) showed data on 101 data sets of individual toxicokinetic parameters for 49 specific substances (mostly drugs) from groups of five or more healthy adults, which suggested that a 10 -fold $\mathrm{UF}_{\mathrm{H}}$ accounted for about $97 \%$ of the variation seen. However, this early work assumed that a $U_{\mathrm{H}}$ of 10 reflected the total range of human variability and not the variability between average and sensitive individuals (see section on interpreting $U_{\mathrm{H}}$ ).

Calabrese (1985) found considerable differences in the capacity of human subjects to metabolize foreign substances, including a 20,000-fold range in abilities to oxidatively metabolize debrisoquine, an antihypertensive drug, and a 10,000-fold range in susceptibilities to vaccine-associated polio among people with hypogammaglobulinemia. Despite those wide variations, the author concluded that the 10-fold factor appeared to provide protection for up to about $80-95 \%$ of the public. Again, however, that conclusion was based on the supposition that $U F_{H}$ is meant to account for the total range of human variability. Actual use of $U_{F}$ suggests that it protects more of the population than this author concluded (see section on interpreting $\mathrm{UF}_{H}$ ).

Some of the studies evaluating intrahuman sensitivity looked separately at the 3.16-fold toxicokinetic and toxicodynamic components of $\mathrm{UF}_{\mathrm{H}}{ }^{12}$ Renwick and Lazarus (1998) investigated a database comprising 60 compounds with metabolism and clearance data and 49 compounds with effects data. The authors found that a kinetic uncertainty factor of $\mathbf{3 . 1 6}$ failed to protect a mean of 0.0685 and $0.8564 \%$ (precision as reported by the authors) of the population, respectively, when a normal or lognormal distribution for the population variability was assumed. A dynamic uncertainty factor of 3.16 failed to protect mean values of 0.2930 and $1.8896 \%$ of the population, respectively. If the kinetic and dynamic aspects of $U F_{H}$ are assumed to be independent, combining both factors to yield the default value of 10 (i.e., $3.16 \times 3.16$ ) protected 99.9998 and $99.9838 \%$ of the population, respectively. However, specific subpopulation comparisons with preterm infants and children or different ethnic groups occasionally lead to greater variation when compared to the standard adult population. Such subpopulation comparisons indicate the

\footnotetext{
12 The value of 3.16 comes from equal subdivision of the default 10-fold $U_{F_{H}}$ into its kinetics and dynamics components. If independent action is assumed, then $3.16 \times 3.16=10$. This factor is also often shown as 3.2 or 3 , because of concerns about portraying uncertainty factors as being overly precise. With any of these designations, however, the intent is to show one-half $\log _{10}$ values. This representation often leads to the somewhat confusing nomenclature found on the EPA's IRIS and elsewhere suggesting that $3 \times 3=10$.
} 
TABLE 3

Summary of U.S. EPA's RfDs on IRIS as of May 2000 Based on Human Data

\begin{tabular}{|c|c|c|c|c|c|c|c|c|c|c|c|c|}
\hline \multirow{2}{*}{$\begin{array}{c}\text { Chemical name } \\
\text { (as on the EPA's IRIS) }\end{array}$} & \multirow[b]{2}{*}{ Specie/type of study } & \multirow{2}{*}{$\begin{array}{l}\text { NOAEL, LOAEL, } \\
\text { or BMD }\end{array}$} & \multirow[b]{2}{*}{ Critical effect(s) } & \multicolumn{7}{|c|}{ Uncertainty factor ${ }^{b}$} & \multirow[b]{2}{*}{$\mathbf{R f D}$} & \multirow{2}{*}{$\begin{array}{c}\text { RfD } \\
\text { confidence }\end{array}$} \\
\hline & & & & Total & $\mathbf{H}$ & A & $\mathbf{L}$ & $\mathbf{s}$ & D I & $\mathbf{M P}$ & & \\
\hline Aldicarb & $\begin{array}{l}\text { Human experimental } \\
\text { gavage }\end{array}$ & 0.01 & $\begin{array}{l}\text { Clinical signs of blood } \\
\text { or plasma cholinesterase } \\
\text { inhibition }\end{array}$ & 10 & 10 & 1 & 1 & 1 & 1 & & $1 E-3$ & Medium \\
\hline Arsenic, inorganic & $\begin{array}{l}\text { Human epidemiology } \\
\text { drinking water }\end{array}$ & 0.0008 & $\begin{array}{l}\text { Skin lesions and possible } \\
\text { vascular complications }\end{array}$ & $\mathbf{3}$ & 3 & 1 & 1 & 1 & 1 & 1 & 3 E-4 & Medium \\
\hline Barium & $\begin{array}{l}\text { Human experimental, } \\
\text { epidemiological } \\
\text { drinking water }\end{array}$ & 0.21 & Increased blood pressure & 3 & 3 & 1 & 1 & & 31 & 1 & $7 \mathrm{E}-2$ & Medium \\
\hline Baygon & $\begin{array}{l}\text { Human experimental } \\
\text { single dose }\end{array}$ & $0.36(L)$ & $\begin{array}{l}\text { Mild cholinergic symptoms, } \\
\text { RBC cholinesterase } \\
\text { inhibition }\end{array}$ & 100 & 10 & 1 & 10 & 1 & 1 & 1 & $4 E-3$ & Medium \\
\hline Benzoic acid & $\begin{array}{l}\text { Human anecdotal } \\
\text { dietary exposure }\end{array}$ & 4.4 & $\begin{array}{l}\text { No adverse effects } \\
\text { observed }\end{array}$ & 1 & 1 & 1 & & & 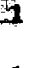 & 1 & $4 \mathrm{E}+0$ & Medium \\
\hline Cadmium & $\begin{array}{l}\text { Human chronic exposures } \\
\text { from a variety of studies }\end{array}$ & 0.005 & Significant proteinuria & 10 & 10 & 1 & 1 & 1 & 1 & 4 & $5 \mathrm{E}-4$ & High \\
\hline Chlorpyrifos & $\begin{array}{l}\text { Human experimental } \\
\text { capsule }\end{array}$ & 0.03 & $\begin{array}{l}\text { Plasma cholinesterase } \\
\text { inhibition }\end{array}$ & 10 & 10 & 1 & 1 & 1 & 1 & & $3 \mathbf{E}-\mathbf{3}$ & Medium \\
\hline $\begin{array}{l}\text { 4,6-Dinitro-o-cyclohexyl } \\
\text { phenol }\end{array}$ & $\begin{array}{l}\text { Human anecdotal } \\
\text { clinical therapy }\end{array}$ & $8.0(\mathrm{LF}$ & Cataract formation & 1000 & 10 & 1 & 10 & 10 & 1 & & $2 \mathbf{E}-\mathbf{3}$ & Low \\
\hline 2,4-Dinitrophenol & $\begin{array}{l}\text { Human anecdotal } \\
\text { clinical therapy }\end{array}$ & $2.0(\mathrm{~L})$ & Cataract formation & 1000 & 10 & 1 & 10 & 10 & 1 & & $2 \mathrm{E}-3$ & Low \\
\hline Ethephon & $\begin{array}{l}\text { Human experimental } \\
\text { oral exposure }\end{array}$ & $0.5(\omega)$ & $\begin{array}{l}\text { Plasma cholinesterase } \\
\text { inhibition }\end{array}$ & 100 & 10 & 1 & 10 & 1 & 1 & & $5 \mathbf{E}-\mathbf{3}$ & Low \\
\hline Ethion & $\begin{array}{l}\text { Human experimental } \\
\text { short term }\end{array}$ & $0.05^{d}$ & $\begin{array}{l}\text { Plasma cholinesterase } \\
\text { inhibition }\end{array}$ & 100 & 10 & 1 & 10 & 1 & 1 & $\mathbf{I}$ & $5 \mathrm{E}-4$ & Medium \\
\hline Fluorine (soluble fluoride) & Human epidemiology & 0.06 & $\begin{array}{l}\text { Objectionable dental } \\
\text { fuorosis in children }\end{array}$ & 1 & 1 & 7 & 1 & 1 & 1 & $\mathbf{1}$ & $6 \mathrm{E}-2$ & High \\
\hline Malathion & $\begin{array}{l}\text { Human experimental } \\
\text { feeding }\end{array}$ & 0.23 & $\begin{array}{l}\text { Erythrocyte cholinesterase } \\
\text { depression }\end{array}$ & 10 & 10 & 1 & 1 & 1 & 7 & & 2 E-2 & Medium \\
\hline Manganese & $\begin{array}{l}\text { Human data of several } \\
\text { types }\end{array}$ & 0.14 & $\begin{array}{l}\text { No LOAEL given, CNS } \\
\text { effects appear to occur } \\
\text { at higher doses }\end{array}$ & & 1 & 3 & 1 & & 4 & 1 & $1.4 \mathrm{E}-3$ & Medium \\
\hline Methylmercury & $\begin{array}{l}\text { Human epidemiological } \\
\text { poisoning }\end{array}$ & $\begin{array}{l}0.000857 \text { to } \\
0.001472(B)\end{array}$ & $\begin{array}{l}\text { Infant developmental } \\
\text { neurological abnormalities }\end{array}$ & 10 & 10 & 1 & & $\mathbf{1}$ & 1 & 1 & $1 E-4$ & High \\
\hline Molybdenum & $\begin{array}{l}\text { Human epidemiological } \\
\text { dietary }\end{array}$ & $0.14(L)$ & Increased uric acid & 30 & 3 & 1 & 10 & 1 & 1 & 1 & $5 \mathbf{E}-\mathbf{3}$ & Medium \\
\hline Nitrate & $\begin{array}{l}\text { Human epidemiology } \\
\text { surveys }\end{array}$ & 1.0 & $\begin{array}{l}\text { Early clinical signs } \\
\text { of methemoglobinemia } \\
\text { in children }<10 \%\end{array}$ & 1 & 1 & 1 & & 1 & 1 & 1 & $1.6 \mathrm{E}+0$ & High \\
\hline Nitrite & $\begin{array}{l}\text { Human epidemiology } \\
\text { surveys }\end{array}$ & 1.0 & $\begin{array}{l}\text { Early clinical signs } \\
\text { of methemoglobinemia } \\
\text { in children }<10 \%\end{array}$ & 1 & 1 & 1 & & 1 & 1 & 10 & $1 \mathrm{E}-1$ & High \\
\hline Pirimiphosmethyl & $\begin{array}{l}\text { Human 56-day } \\
\text { experimental feeding }\end{array}$ & 0.25 & $\begin{array}{l}\text { Transient plasma } \\
\text { cholinesterase inhibition }\end{array}$ & 25 & 10 & 1 & 2.6 & 1 & 1 & 1 & $1 \mathrm{E}-2$ & High \\
\hline Selenium and compounds & $\begin{array}{l}\text { Human food and } \\
\text { soil epidemiology }\end{array}$ & 0.016 & Clinical selenosis & 3 & 3 & 1 & 1. & 3 & 1 & & $5 \mathrm{E}-3$ & High \\
\hline Silver & $\begin{array}{l}\text { Human anecdotal } \\
\text { studies }\end{array}$ & 0.014 & Arguria & 3 & 3 & 1 & 1 & 1 & 1 & 1 & $5 E-3$ & Low \\
\hline $\begin{array}{l}\text { 1,1,2-Trichloro- } \\
\text { 1,2,2-trifluoroethane }\end{array}$ & $\begin{array}{l}\text { Human occupational } \\
\text { exposure }\end{array}$ & 273 & Psychomotor impairment & 10 & 10 & 1 & $\mathbf{1}$ & 1 & 1 & 1 & $3 \mathbf{E}+1$ & Low \\
\hline Warfarin & Human experimental & 0.020 & Increased prothrombin time & 100 & 10 & 1 & 10 & 1 & 1 & 1 & $3 E-4$ & Low \\
\hline Zinc and compounds & $\begin{array}{l}\text { Human experimental } \\
\text { diet supplement }\end{array}$ & $69.7(L)$ & $\begin{array}{l}\text { Decrease in erythrocyte } \\
\text { superaxide dismutase } \\
\text { concentration in adults }\end{array}$ & $\mathbf{3}$ & 1 & 1 & $\mathbf{3}$ & 1 & 1 & $1]$ & $3 \mathbf{E}-1$ & Medium \\
\hline
\end{tabular}

a All values are in mg/kg-day and are NOAELs unless otherwise stated: (L), LOAEL; (B), benchmark dose (BMD).

${ }^{b}$ Uncertainty factors are $H$, average human to sensitive human; $A$, animal to human; L, LOAEL to NOAEL; S, subchronic exposure to chronic; D, database insufficiency; MF, modifying factor to account for uncertainties not covered by the traditional factors.

c Based on analogy to 2,4-dinitrophenol.

'The EPA's IRIS lists this value as a NOEL, but also adds an uncertainty factor for LOAEL to NOAEL extrapolation because of the proximity of this NOEL to brain cholinesterase inhibition in dogs at $0.71 \mathrm{mg} / \mathrm{kg}$-day

e Based on the toxicity of nitrate with an adjustment to this dose with a 10-fold MF. 
TABLE 4

Comparison of Different Investigators' Estimations of Protectiveness of the Intraspecies Uncertainty Factor $\left(\mathbf{U F}_{\mathrm{H}}\right)$,

Based on Different Data Sets and Starting Points

\begin{tabular}{|c|c|c|c|c|}
\hline Investigators & $\begin{array}{l}\text { Percentage of chemicals } \\
\text { or population protected by } \\
\text { UF }_{\mathrm{H}} \text { of } 10 \text { or subfactor of } 3.16\end{array}$ & $\begin{array}{l}\mathrm{UF}_{\mathrm{H}} \text { for } 99 \% \\
\text { protection }\end{array}$ & Starting point & Data set and comments \\
\hline \multicolumn{5}{|c|}{ Variability among adults } \\
\hline Dourson and Stara, 1983 & $\begin{array}{l}\text { For } 92 \% \text { of the chemicals } \\
\text { tested, } 99.9 \% \text { of the } \\
\text { population protected }\end{array}$ & 6 & $\begin{array}{l}\text { Animal } \mathrm{LD}_{50} \text { analysis of } \log \\
\text { probit slopes }\end{array}$ & $\begin{array}{l}\text { Assumes that } \mathrm{UF}_{\mathrm{H}} \text { of } 10 \text { covers from the average to the } \\
\text { sensitive human response. }\end{array}$ \\
\hline Brown, 2001 & $\begin{array}{l}97 \% \text { protection of the } 5 \text { th } \\
\text { percentile of a } \\
\text { compromised population }\end{array}$ & Not estimated & $\begin{array}{l}\mathrm{ED}_{50} \text { or } \mathrm{LD}_{50} \text { of the normal } \\
\text { or compromised population } \\
\text { compared }\end{array}$ & Interhuman variability in critically ill or injured subjects. \\
\hline Hattis et al., 1999a,b & $\begin{array}{l}99.99 \text { to } 99.9 \% \text { of the } \\
\text { population }\end{array}$ & Not estimated & $\begin{array}{l}5 \% \text { response level in a } \\
\text { normal population }\end{array}$ & $\begin{array}{l}\text { Based on assumption that population sensitivity is lognormal } \\
\text { out to extreme tails; data include parameters related to } \\
\text { health risks in adults and some children. }\end{array}$ \\
\hline Brock, 1991 & $99.97 \%$ of the population & Not estimated & $\begin{array}{l}\text { Human variation in } \\
\text { cholinesterase inhibition } \\
\text { among and within } \\
\text { individuals }\end{array}$ & $\begin{array}{l}\text { Comparison given here is for a specific dynamic parameter. The } \\
\text { proper comparison of these ratios is to the dynamic factor of } \\
3.16 \text { for } \mathrm{UF}_{\mathrm{H}} \text {. }\end{array}$ \\
\hline Hattis et al., 1987 & $97 \%$ of the population & 15 & $\begin{array}{l}\text { Human kinetic } \\
\text { parameters }\end{array}$ & $\begin{array}{l}\text { Based on toxicokinetic parameters in healthy adults. Assumed } \\
\text { that } U F_{H} \text { of } 10 \text { covered the whole range of the human } \\
\text { population. This early work did not separately evaluate the } \\
\text { kinetic subpart of } 3.16 \text { for } U F_{H} \text {. }\end{array}$ \\
\hline Calabrese, 1985 & $\begin{array}{l}80 \text { to } 95 \% \text { of the } \\
\text { population }\end{array}$ & Not estimated & None given & $\begin{array}{l}\text { Comparisons are for both effects and specific kinetic or dynamic } \\
\text { parameters. Thus, the proper comparison of these ratios } \\
\text { might be to either the full } 10 \text {-fold } \mathrm{UF}_{\mathrm{H}} \text { or to the } 3.16 \text {-fold } \\
\text { components, which makes the interpretation of this study } \\
\text { difficult. Furthermore, the author assumed that } U F_{H} \text { of } 10 \\
\text { covered the whole range of the human population (see text for } \\
\text { a discussion of this error). }\end{array}$ \\
\hline $\begin{array}{l}\text { Renwick and Lazarus, } \\
1998\end{array}$ & $\begin{array}{l}99.9998 \text { to } 99.9838 \% \text { of the } \\
\text { population }\end{array}$ & Not estimated & $\begin{array}{l}\text { Analysis of variability in } \\
\text { kinetic and dynamic } \\
\text { parameters }\end{array}$ & $\begin{array}{l}\text { Assumes that the kinetic and dynamic aspects of } \mathrm{UF}_{H} \text { are } \\
\text { independent; children do not appear, in general, to represent } \\
\text { a sensitive subgroup from the kinetic aspects; genetic } \\
\text { polymorphisms or ethnic differences may decrease the overall } \\
\text { proportion of the population protected by a UF } \mathrm{UF}_{H} \text { of } 10 \text {. }\end{array}$ \\
\hline Renwick et al., 2001 & $99.9 \%$ of the population & 24 & $\begin{array}{l}\text { Human variability in kinetic } \\
\text { parameters associated } \\
\text { with CYP1A2 metabolism }\end{array}$ & $\begin{array}{l}\text { Analysis based on } 3.16 \text { kinetic part of } \mathrm{UF}_{\mathrm{H}} \text { in healthy adult } \\
\text { populations. }\end{array}$ \\
\hline Silverman et al., 1999 & $\begin{array}{l}\text { For } 80 \% \text { of chemical-specific } \\
\text { kinetic parameters, } 96 \text { to } \\
99.999 \% \text { of the population } \\
\text { protected }\end{array}$ & Not estimated & $\begin{array}{l}\text { Human kinetic default } \\
\text { uncertainty factor of } 3.2\end{array}$ & $\begin{array}{l}\text { Human kinetic variability (i.e., AUC and } C_{\max } \text { in response to } 6 \\
\text { drugs; some information on children available but } \\
\text { insufficient for separate conclusions. }\end{array}$ \\
\hline \multicolumn{5}{|c|}{ Variability between children and adults } \\
\hline Glaubiger et al., 1982 & $100 \%$ of the chemicals & Not estimated & $\begin{array}{l}\text { Human MTDs of the } \\
\text { anticancer drugs in } \\
\text { children and adults }\end{array}$ & $\begin{array}{l}\text { Study was not designed to test the adequacy of } \mathrm{UF}_{\mathrm{H}} \text {; mean } \\
\text { values do not show distributions of sensitivities between } \\
\text { groups. }\end{array}$ \\
\hline $\begin{array}{l}\text { NAS, 1983; Charnley and } \\
\text { Putzrath, } 2001\end{array}$ & Not estimated & Not estimated & $\begin{array}{l}\text { Animal comparisons of } \\
\text { chemically induced } \\
\text { carcinogenesis based on } \\
\text { age }\end{array}$ & $\begin{array}{l}\text { Younger animals are less susceptible than adults for } 47 \% \text { of the } \\
\text { time, equally sensitive for } 13 \% \text {, and more sensitive for } 40 \% \text {. }\end{array}$ \\
\hline
\end{tabular}


Sheehan and Gaylor,

1990

Calabrese, 2001

Rane, 1992

Renwick, 1998

Naumann, 2001

Skowronski and

Abdel-Rahman,

2001

Ginsberg et al., 2002
$86 \%$ of the chemicals

$86 \%$ of the chemicals

$67 \%$ of the chemicals

$91 \%$ of the chemicals

All values are for \%

of the population

Antidepressants

Dynamics: 100

Kinetics: 92

Angiotensin inhibitors

Dynamics: 100

Kinetics: 96

Nonsteroidal

antiinflammatory

Dynamics: NE

Kinetics: 60

Cholesterol-lowering drugs

Dynamics: NE

Kinetics: 86

Antibiotics

Dynamics: NE

Kinetics: 90

$100 \%$ of 6 drugs studied Not estimated

Not estimated, but would vary among different

drugs studied and age of

the child.
Not estimated

Not estimated

Not estimated

Not estimated

Not estimated

$\mathrm{NE}$, not estimated

Notimated

Not estimated to younger

Animal $L_{50} 8$ ratios of adult to younger

Human newborn and adult clearance values compared

Human infant and child and adult clearance and elimination compared

Human kinetic and dynamic values compared among different subpopulations including aged, children, normal, and those with advanced disease
Data are from 238 chemicals, but information given in an abstract only.

Data are from 313 chemicals in 25 chemical classes.

Analysis besed on 3.16 kinetic part of $\mathrm{UF}_{\mathrm{H}}$

Analysis based on 3.16 kinetic part of $U F_{H}$.

Data are from five classes of drugs. Subfactors for dynamics and kinetics were not combined as in Renwick and Lazarus (1998) shown above, and thus the protectiveness of the overall default value of 10 for $\mathrm{UF}_{\mathrm{H}}$ is not evaluated by these investigators.
Toxicokinetic factors compared among children, adults, and elderly

Human variability in

toxicokinetics for $\mathbf{4 5}$ drugs; kinetic default uncertainty factor of 3.16 was analyzed.
Authors analyzed the composite factor of 10 using comparative kinetic data and the IPCS default value for toxicodynamic variability. All estimated composite values were less than 10 . Study was specifically designed to compare adult and child

toxicokinetic parameters. Several different stages of childhood were studied. Premature and full-term neonates and newborns have a statistically significant higher average half-life and correspondingly lower average clearance when compared with adult values. Older children have statistically significant higher average clearances. Age-specific

adjustment factors were recommended when sufficient data on a chemical are available. 
need for caution in stating the protectiveness of default uncertainty factors and suggest the need for additional research on polymorphisms as they relate to population variability in toxic response. Renwick et al. (2001) also studied human variability in kinetic parameters associated with CYP1A2 metabolism, finding differences within healthy populations of 1.8 or 2.4 for the 95 th or 99th percentiles, respectively.

Silverman et al. (1999) looked at intrahuman variability in pharmacokinetic parameters from published pharmaceutical clinical trial data for six compounds. Specifically, the authors examined the area under the chemical concentration-time curve (AUC) and the peak plasma concentration $\left(C_{\max }\right)$. The purpose of their work was to investigate the use of specific data describing intrahuman variability in lieu of the usual default $U_{F}$ value of 10 . Review of the data indicated that the default value of 3.2 for the kinetic component of $\mathrm{UF}_{\mathrm{H}}$ was more than sufficient in 12 of 15 cases. For those cases, a relatively small percentage of the population $(0.001$ to $3.8 \%)$ would be left unprotected if kinetic data were substituted for the default kinetic value and combined with the default value of 3.2 for the dynamic component of $\mathrm{UF}_{\mathrm{H}}$. In the remaining 3 instances, a composite factor greater than the usual default $\mathrm{UF}_{\mathrm{H}}$ value of 10 would be required to provide adequate coverage (defined by the authors as $95 \%$ of the sensitive population).

\section{Adequacy of $U F_{H}$ for Children}

Although the studies described above indicate that a 10-fold $U_{F}$ generally protects individuals with greater-than-average sensitivities, recent concern has focused on the adequacy of $\mathrm{UF}_{\mathrm{H}}$ for protecting children in particular. This concern has prompted evaluations of the differences in susceptibility to chemical toxicity between younger animals and older animals and of differences in clinical sensitivities to pharmaceutical agents in children compared to adults for a variety of agents. The studies discussed here represent the few that permit quantitative evaluation of age-related differences in sensitivity for the purpose of evaluating the adequacy of $\mathrm{UF}_{\mathrm{H}}$ for children. These studies are also summarized in Table 4 and described below.

Glaubiger et al. (1982) compared maximum tolerated doses (MTDs) ${ }^{13}$ for 17 anticancer drugs in children and adults. Ratios of the child MTD to the adult MTD, when measured as milligrams per square meter of surface area or milligrams per kilogram per day, varied from 0.83 to greater than 2.2. Three ratios were less than 1 ;

\footnotetext{
${ }^{13}$ It should be noted here and with previous and subsequent discussion of $L D_{50}$ 's that such high-dose studies invariably use gavage or bolus dosing, resulting in peak loads that can saturate detoxification mechanisms in newborns. Similar dases given at rates that simulate environmental exposure might be detoxified more efficiently, and the values of adult to younger human or animal MTDs or $\mathrm{LD}_{50}$ 's might be more similar.
}

1 ratio equaled 1; and 13 ratios were greater than 1. Ratios greater than 1 indicated that the child was less sensitive than the adult. The mean ratio was at least 1.3. However, if all of the ratios were based on milligram per kilogram per day doses, which is the dose measurement on which uncertainty factors are based, the ratios varied from about 1.3 to over 4.1 , with a mean ratio of 2.3. As before, ratios greater than 1 indicate that children are less sensitive than adults to the toxicity of these chemicals. Because these ratios are for an effect, not for a specific kinetic or dynamic parameter as discussed elsewhere in this text, the proper comparison of the ratios is to the full 10 -fold $\mathrm{UF}_{\mathrm{H}}$. Thus, for each of the 17 chemicals evaluated, children are less sensitive than adults on a milligram per kilogram per day basis, although the overall difference in sensitivity between children and adults is quite small as measured by mean ratios.

The National Academy of Sciences report Pesticides in the Diets of Infants and Children (NAS, 1983) included a table summarizing the results of studies that had been performed through 1983 in which the effects of age on chemically induced carcinogenesis in rodents had been evaluated. Charnley and Putzrath (2001) updated those results to include studies performed since 1983. The data indicate that there are a similar number of studies demonstrating that younger animals are less susceptible than adults $(47 \%)$ to chemically induced carcinogenesis as there are demonstrating that they are more susceptible (40\%) under the conditions of the bioassays. A number of studies showed that age played no role at all in susceptibility (13\%). The extent of the age-related differences was not evaluated quantitatively because virtually all of the studies were performed using only one high dose level, so the underlying dose-response relationships are unknown. The NAS report concluded that those results clearly demonstrate that age may be an important factor in susceptibility to chemically induced carcinogenesis, but they do not support the conclusion that younger animals are always more susceptible than older animals. The NAS went on to further conclude that $\mathrm{UF}_{\mathrm{H}}$ of 10-fold provides adequate protection of infants and children, based on current knowledge (Bruckner, 2000).

Sheehan and Gaylor (1990) compared the $\mathrm{LD}_{50}$ ratios of adult to newborn mammals for 238 chemicals as a measure of intraspecies variability. The median ratio was 2.6 (adult to newborn). In contrast to Glaubiger et al. (1982) above, ratios greater than 1.0 indicate that the child is more sensitive than the adult. The percentage of $L D_{50}$ ratios less than 10 was $\sim 86 \%$. Because the ratios given here are for an effect and not a specific kinetic or dynamic parameter, the proper comparison is to the full 10 -fold $U F_{H}$. Thus, for the chemicals evaluated, young animals on average were more sensitive than adults to acute lethality, and the 10 -fold $\mathrm{UF}_{\mathrm{H}}$ would 
adjust the adult animal $L D_{50}$ to that of the younger animals for $86 \%$ of the chemicals tested.

Calabrese (2001) showed a similar analysis of age comparisons for $\mathrm{LD}_{50}$ determinations in laboratory animals for 313 chemicals. Ninety-seven adult to young $\mathrm{LD}_{50}$ ratios $(31 \%)$ were between 0.5 and less than 2.0 , indicating that adults and young animals were equally sensitive. Adults displayed greater sensitivity in 46 cases (14\%), exceeding 10 -fold greater sensitivity than younger animals in 4 cases (1\%). Younger animals displayed greater sensitivity in 170 cases $(54 \%)$, exceeding 10-fold greater sensitivity than older animals in 43 cases (14\%). Again, young animals were found on average to be more sensitive than adults to acute lethality and the full 10-fold $\mathrm{UF}_{\mathrm{H}}$ would adjust the adult animal $\mathrm{LD}_{50}$ to that of the younger animal for $86 \%$ of the chemicals tested.

Rane (1992) compared the elimination half-lives in newborn and adult humans for 14 drugs of known hepatic clearance. He illustrated that newborns were poorer at clearance when compared with adults for the majority of chemicals studied (i.e., 10 of 14) and thus presumably would be more sensitive to their toxicity; that is, newborns were exposed to a larger internal dose of those chemicals than adults. The ratios of newborn to adult kinetic parameters varied from 0.60 to $17 ;^{14}$ ratios greater than 1.0 suggest that the child was more sensitive than the adult. The arithmetic average was 3.5. Because the comparison given by Rane (1992) is for a specific kinetic parameter and not for an effect, as discussed in each of the three studies on $\mathrm{LD}_{50}$ or MTD, the proper comparison for these ratios is to the 3.16 kinetic component of the $\mathrm{UF}_{\mathrm{H}}$. On that basis the newborn is more sensitive on average than the adult (i.e., the average ratio is greater than 1.0), but the 3.16 kinetic component of $U F_{H}$ would adjust the adult human clearance value to that of the newborn for $71 \%$ of the chemicals studied (i.e., $71 \%$ of the ratios are less than 3.2, which is the multiple of 1.0 and 3.16). Rane (1992) also compared the half-lives of drugs that were unclassified with respect to hepatic clearance in newborn and adult humans. The mean ratio of newborn to adult kinetic parameters was 7.8 , but the 3.16 kinetic component of $\mathrm{UF}_{\mathrm{H}}$ adjusted the adult human clearance value to that of the newborn for $60 \%$ of these chemicals. The composite percentage for both groups of chemicals is $67 \%$.

Renwick (1998) discussed toxicokinetics, principally clearance and elimination half-time, in infants and children in relation to adults and evaluated whether $\mathrm{UF}_{\mathrm{H}}$ is sufficiently large, in general, to afford protection to children. In contrast to what Rane demonstrated for newborns, Renwick (1998) illustrated that infants and

\footnotetext{
14 These ratios are estimated based on the data provided by Rane (1992). Two digits of precision to these ratios are specified despite the fact that not all data provided by Rane had this level of precision.
}

children have more rapid clearance rates when compared with adults for the majority of chemicals studied (16 of 22) and concluded that infants and children are, therefore, exposed to lower doses of those chemicals than adults. The arithmetic average ratio of infant or child to adult kinetic parameters was 1.8 as determined by us using the data provided by Renwick (1998); ratios greater than 1.0 suggest that the infant or child is less sensitive than the adult because clearances (generally) are being compared. [In contrast, Rane (1992) compared half-lives.] Moreover, when infants or children eliminated chemicals more slowly than adults, that difference was no greater than 5-fold based on the mean ratios of infant or child to adult kinetic parameters (see Fig. 7). Variations within the adult and infant or child subgroups, when given, appear to be similar. Renwick concluded that the higher clearance of many xenobiotics by children compared with adults may compensate, at least in part, for increased organ sensitivity during development and that an increased uncertainty factor for postsuckling infants and children is not required. As above, because the comparison given by Renwick is for a specific kinetic parameter and not for an effect, the proper comparison of these ratios is to the 3.16 kinetic component of $\mathrm{UF}_{\mathrm{H}}$. On that basis, infants and children on average are less sensitive than adults to chemical toxicity (i.e., the average ratio is greater than 1.0), but when they are not, the 3.16 kinetic component of $U_{F}$ adjusts the adult human kinetic parameter to that of the infant or child for $91 \%$ of the chemicals tested (i.e.,

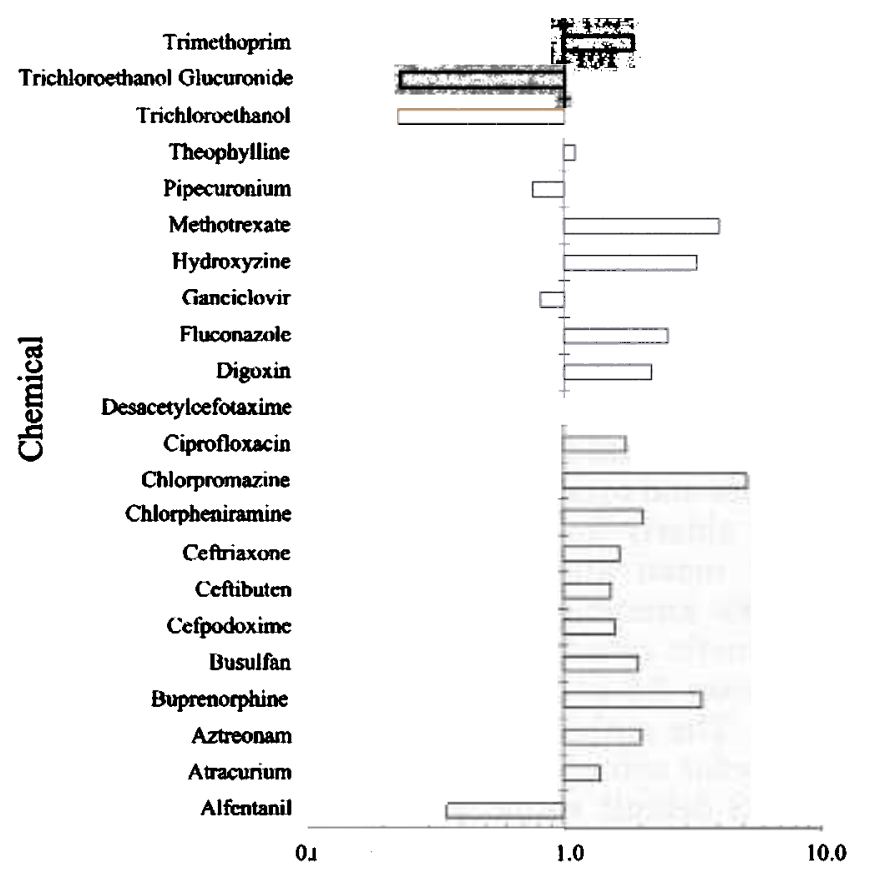

Ratio of Child to Adult Value (1 Indicates Unity)

FIG. 7. Mean ratios of child to adult selected kinetic parameters (based on data from Renwick, 1998). 
$91 \%$ of the ratios are greater than 0.32 , which is the division of a ratio of 1.0 by 3.16 ). Renwick et al. (2000) extended this work by analyzing chemicals that are eliminated primarily by a single pathway and reached similar conclusions.

Naumann (2001) investigated the adequacy of the 3.16 $\mathrm{UF}_{\mathrm{H}}$ subfactors using representative chemicals from five different therapeutic classes and different human populations. These populations included diseased individuals and some children. Subfactors were calculated as either the ratio of the mean and 2 standard deviations from a normal population for selected toxicodynamic and toxicokinetic parameters or the ratio of the mean of a normal population and the lower $95 \%$ of defined (by data) sensitive populations. For antidepressants these authors found that all toxicodynamic ratios varied between 1.1 and 1.7 while toxicokinetic ratios varied between 1.2 and 2.6 for normal populations and 2.9 to 7.5 for sensitive subpopulations. Of the 36 ratios studied for the latter group, $33(92 \%)$ were less than the 3.16 kinetic component of $\mathrm{UF}_{\mathrm{H}}$. For angiotensin inhibitors, all ratios were between 1.06 and 1.89 for dynamic variability while toxicokinetic ratios varied from 1.09 to $6.15 ; 149$ of 156 ratios $(96 \%)$ were less than the 3.16 kinetic component of $U F_{H}$. For nonsteroidal antiinflammatory drugs, toxicodynamic ratios were not determinable. Toxicokinetic ratios varied from 1.19 to 2.86 for the healthy population, while for several classes of sensitive individuals, ratios varied between 0.15 and 11.82. Of 53 ratios studied, $32(60 \%)$ were less than 3.16. For cholesterol-lowering drugs, toxicodynamic ratios were not determinable. Toxicokinetic ratios varied from 1.3 to 2.2 for healthy populations and from 0.9 to 6.4 for various subpopulations including sensitive groups. Of 28 ratios evaluated, 24 (86\%) were less than 3.16. For antibiotics, toxicodynamic ratios were not determinable. Toxicokinetic ratios varied from 1.3 to 4.7 ; of 10 ratios studied, $9(90 \%)$ were less than 3.16 . Thus, the default value of 3.16 for both toxicodynamics and toxicokinetics accounts for population variability most of the time, and these populations include diseased individuals.

Skowronski and Abdel-Rahman (2001) compared toxicokinetics and toxicodynamics among children, adults, and the elderly for six drugs. They estimated ratios of adult mean kinetic parameters to corresponding lower $95 \%$ kinetic values for children and the elderly. Toxicokinetic ratios between children and adults varied between 0.6 and 3.7 ; the lone toxicodynamic ratio was 1.2. The authors estimated the composite uncertainty factor using these comparative kinetic data and the IPCS default value for toxicodynamic variability. All composite values were less than a default value of 10-fold.

Ginsberg et al. (2002) evaluated child to adult toxicokinetics differences from a database derived from the therapeutic literature. The database was robust enough to demonstrate comparisons between adults and children of differing ages, for example, premature and fullterm neonates, newborns ( 1 week to 2 months), and early infants ( 2 to 6 months). The database consisted of information on a number of cytochrome P450 pathways, certain phase II conjugation reactions, and renal elimination for $\mathbf{4 5}$ drugs. Results indicated that premature infants had on average about a four-fold longer half-life than adults. For full-term neonates and newborns, this average difference was about two-fold. Differences in average clearance were somewhat smaller among these groups, being less than two-fold in all cases. After 6 months, half-lives for these drugs in children were often found to be shorter than corresponding half-lives in adults; clearances were correspondingly higher. Half-lives and clearances were more variable for different types of chemicals. For example, for the CYP1A2 substrates caffeine and theophylline, the average half-life was approximately nine-fold greater for full-term neonates and about four-fold for newborns when compared with adult values.

\section{DISCUSSION}

Questions have been raised about whether current regulatory approaches to limiting chemical exposures are adequate to protect children from toxicity. Lead is often cited as an example of failed regulation due to insensitive animal testing. However, if lead were regulated on the basis of its developmental toxicity in laboratory animals, its action level would be much lower than it is at present, even without the addition of a child-protective safety factor (Plunkett, 1999).

Laboratory animals can be useful predictors of chemical hazards to humans whether they pose threats to children or adults. As more fully discussed in Scheuplein et al. (2002), growth and development are compressed into a shorter period in animals, which makes animal testing inherently more difficult. However, similar developmental events occur in both humans and laboratory animals and testing that covers the full period of animal development can reasonably be considered an appropriate surrogate for human development. It is likely that the weakest systems in the animal will be challenged sufficiently by the high doses tested to compensate adequately for compressed exposure and maturation periods. As long as the toxicity testing framework includes studies that encompass the developmental period and include systemic toxicity endpoints as appropriate, there is no reason to expect that significant human health hazards would not be detected by appropriate animal models. Overall, there appears to be a reasonable concordance among species for developmental toxicants and appropriate protocols compensate for differences in maturation rates. Additionally, testing guidelines appear to capture adequately a significant proportion of potential critical 
effects, and tiered systems of testing offer increasingly detailed data sets that provide information on the developing animal. Furthermore, although the data available to demonstrate the adequacy of $\mathrm{UF}_{\mathrm{D}}$ are not extensive, studies suggest that their use protects against the likelihood that toxicity occurs at a lower dose or for another effect than those tested most of the time.

When the aim is to identify and rank chemicals as potential developmental threats in order to establish priorities for further testing, various developmental toxicology screening protocols are available and should be used. Comprehensive developmental toxicity testing of all chemicals is unnecessary (e.g., inhalation exposure of hydrochloric acid). Consideration of screening test results and the likely nature and extent of exposures should identify substances that need further testing. When more detailed knowledge of potential hazard is needed, current toxicity testing protocols adequately detect potential developmental toxicants as long as studies are conducted in animal models that cover the full developmental period in humans and the appropriate endpoints are measured.

Testing guidance has evolved over the past 2 decades and, at present, approaches to hazard characterization include in utero exposures. Evaluation of results from well-designed and conducted developmental toxicity, reproductive toxicity, and longer term repeat-dose studies and integration of those results with appropriate uncertainty factors is a reasonable and appropriate sciencebased approach to limiting risks to the developing human.

The EPA risk assessments include the use of uncertainty factors when identifying criteria for limiting chemical exposures. Those factors are designed to account for differences in susceptibility within and among species and to compensate for limited data availability, when necessary. Proposals have been made to use an additional 10-fold uncertainty factor for the extra protection of children when estimating safe exposure limits from a database that is inadequate to determine whether children are more sensitive to a chemical's toxicity than adults. Use of such an additional uncertainty factor, as is currently stated by the Food Quality Protection Act (FQPA) for pesticide safety evaluations, is meant to address the same issues already addressed by the EPA's database uncertainty factor, $\mathrm{UF}_{\mathrm{D}}$, with additional issues related to exposure uncertainty. The EPA further states that the use of the FQPA factor should be modified when $U_{D}$ has already been used (U.S. EPA, 2002; Fenner-Crisp, 2001). Based on the data presented here, the authors agree with this position.

Drawing conclusions about the adequacy of $\mathrm{UF}_{\mathrm{H}}$, the uncertainty factor used to account for intrahuman variability, in terms of its ability to protect children from environmental chemical exposures on the basis of the modest data available is somewhat challenging. However, the studies reviewed here suggest that $U_{H}$ pro- tects sensitive groups most of the time. Virtually all of the studies available suggest that a high percentage of the population, including children, is protected by using a 10-fold uncertainty factor for human variability or by using a 3.16-fold factor for either toxicokinetic or toxicodynamic variability (Table 4). Based on specific comparisons for newborns, infants, children, and adults, the percentage of the population protected is between 67 and 100 , with the studies in larger populations that include sensitive individuals suggesting that the value is closer to $100 \%$. That percentage can be as low as 60 for other sensitive populations, including those with severe disease.

Where available, quantitative analysis of the extent of toxicodynamic and toxicokinetic variability among humans indicates that relying on a default value of 10 to compensate for variability among humans, including that due to age, and on a default value of 10 to compensate for a limited toxicity database, when necessary, is adequate to protect most of the people-including children-most of the time. Perhaps the strongest studies from which to draw reasonable and general conclusions are those of Renwick and Lazarus (1998) and Hattis et al. (1999a,b). Both groups of investigators worked from large databases that included both kinetic and dynamic parameters and evaluated normal and sensitive populations, including children (Table 4). The conclusion of both groups is that a $\mathrm{UF}_{\mathrm{H}}$ of 10 is likely to protect $99.9 \%$ or more of the population, and this population includes children. Because $\mathrm{UF}_{\mathrm{H}}$ is applied to a value in the low end of the distribution of human sensitivities (i.e., a NOAEL), its use actually covers total human sensitivity variations of 100 to 1000 times and not 10 times, as is often thought. Furthermore, while our presumption that $\mathrm{UF}_{\mathrm{D}}$ and $\mathrm{UF}_{\mathrm{H}}$ are independent seems reasonable, this presumption may not be reasonable for the two $\mathrm{UF}_{H}$ subfactors. If this assumption is not reasonable, then multiplying factors together, as is commonly done, is likely to introduce added conservatism. Thought about in that context, the combined likelihood that $U F_{H}$ and $U F_{D}$ are adequate to protect children from unanticipated chemical toxicity appears very probable.

Taken together, information on the relative sensitivities of children and adults, on the sensitivity and specificity of toxicity testing protocols, and on the extent to which current uncertainty factors compensate for increased sensitivities and limited data suggests that the use of additional uncertainty factors to limit environmental chemical exposures is unlikely to provide significantly greater protection to children over 6 months of age. The same conclusion might not always hold true for children younger than 6 months of age in the absence of adequate developmental or systemic toxicity testing. However, while younger children are often more sensitive to toxicity than older children or adults, so are younger laboratory animals. Thus, appropriate in utero 
and early neonatal toxicity testing will compensate for any additional early sensitivity. Developmental and reproductive toxicity testing protocols such as those recommended by the EPA, FDA, and OECD are useful for characterizing toxicity in developing animals and for assessing risks to children that might arise from in utero and postnatal exposures.

\section{ACKNOWLEDGMENTS}

The authors acknowledge the support of CropLife America and the American Chemistry Council in part for the development of this work. The comments of Dr. Susan Felter of Proctor and Gamble and Dr. Lynne Haber of Toxicology Excellence for Risk Assessment were also invaluable in the development of key issues. The authors also thank Meg Poehlmann and Sheri Lawson for their excellent assistance in manuscript preparation.

\section{REFERENCES}

Baird, J. S., et al. (1996). Noncancer risk assessment: Probabilistic characterization of population threshold doses. HERA 2, 79-102.

Barnes, D. G., and Dourson, M. L. (1988). Reference dose (RfD): Description and use in health risk assessments. Regul. Toxicol. Pharmacol. 8, 471-486.

Brock, A. (1991). Inter and intraindividual variations in plasma cholinesterase activity and substance concentration in employees of an organophosphorus insecticide factory. Br. J. Ind. Med. 48(8), 562-567.

Brown, D. R. (2001). Risk assessment around the country: A forum for risk assessment information and analysis developed to inform regional, state, and local policy decisions. HERA 7(1), 209-213.

Bruckner, J. V. (2000). Differences in sensitivity of children and adults to chemical toxicity: The NAS panel report. Regul. Toxicol. Pharmacol. 31(3), 280-285.

Burin, G. J., and Saunders, D. R. (1999). Addressing human variability in risk assessment-The robustness of the intraspecies uncertainty factor. Regul. Toxicol. Pharmacal. 30, 209-216.

Calabrese, E. J. (1985). Uncertainty factors and interindividual variation. Regul. Toxicol. Pharmacol. 5(2), 190-196.

Calabrese, E. J. (2001), Assessing the default assumption that children are always at risk. HERA $7(1), 37-59$.

Charnley, G., and Putzrath, R. M. (2001). Children's health, susceptibility, and regulatory approaches to reducing risks from chemical carcinogens. Environ. Health Perspect. 109(2), 187-192.

Christian, M. S. (1986). A critical review of multigeneration studies. J. Am. Coll. Toxicol. 5(2), 161-180.

Clegg, D. J. (1978). Toxicology basis of the ADI-Present and future considerations. In Pesticide Reviews (H. Frehse and H. Geissbuhler, Eds.). Pergamon, Oxford.

Dourson, M. L., and Stara, J. F. (1983). Regulatory history and experimental support of uncertainty (safety) factors. Regul. Toxicol. Pharmacol. 3(3), 224-238.

Dourson, M. L. (1994). Methods for establishing oral reference doses. In Risk Assessment of Essential Methods (Mertz, Abernathy, and S. S. Olin, Eds.), pp. 51-61. ILSI Press: Washington, DC.

Dourson, M. L., et al. (1996). Evolution of science-based uncertainty factors in noncancer risk assessment. Regul. Toxicol. Pharmacol. 24(2), 108-120.

Dourson, M. L., et al. (1992). On reference dose (RfD) and its underlying toxicity data base. Tbxicol. Ind. Health 8(3), 171-189.
Fenner-Crisp, P. (2001). The FQPA 10x safety factor-How much is science? How much is sociology? HERA 7(1), 107-116.

Francis, E. Z., et al. (1990). Workshop on the qualitative and quantitative comparability of human and animal developmental neurotoxicity: Summary and implications. Neurotoxicol. Teratol. 12(3), 285-292.

Ginsberg, G., et al. (2002). Evaluation of child/adult pharmacokinetic differences from a database derived from the therapeutic drug literature. Toxicol. Sci. 66(2), 185-200.

Glaubiger, D. L., et al. (1982). The relative tolerance of children and adults to anticancer drugs. Front. Radiat. Ther. Oncol. 16, 42-49.

Grandjean, P. (1992). Individual susceptibility to toxicity. Toxicol. Lett. 64-65 (5 Pt.1), 43-51.

Hattis, D., el al. (1987). Human variability in susceptibility to toxic chemicals-A preliminary analysis of pharmacokinetic data from normal volunteers. Risk Anal. 7(4), 415-426.

Hattis, D., et al. (1999a). Distributions of individual susceptibility among humans for toxic effects. How much protection does the traditional tenfold factor provide for what fraction of which kinds of chemicals and effects? Ann. N. Y. Acad. Sci. 895(1), 286-316.

Hattis, D., et al. (1999b). Human interindividual variability in parameters related to health risks. Risk Anal. 19(4), 711-726.

Hemminki, K, and Vineis, P. (1985). Extrapolation of the evidence on teratogenicity of chemicals between humans and experimental animals: chemicals other than drugs. Teratog. Carcinog. Mutagen. 5(4), $251-318$.

Hill, A. B. (1965). The environment and disease: Association or causation? Proc. $R$. Soc. Med. 58, 295-300.

Himwich, W. A. (1973). Problems in interpreting neurochemical changes occurring in developing and aging animals. Prog. Brain Res. 40, 13-23.

IPCS. (1994). International Programme on Chemical Safety. Environmental Health Criteria 170: Assessing Human Health Risks of Chemicals: Derivation of Guidance Values for Health-Based Exposure Limits. World Health Organization, Geneva.

Jarabek, A. M. (1994). Inhalation RfC methodology: Dosimetric adjustments and dose-response estimation of noncancer toxicity in the upper respiratory tract. Inhal. Toxicol. 6(Suppl), 301-325.

Jarabek, A. M. (1995). The application of dosimetry models to identify key processes and parameters for default dose response assessment approaches. Toxicol. Lett. 79, 171-184.

Kalberlah, F., and Schneider, K. (1998). Quantification of Extrapolation Factors. Final report of Research Project No. 11606 113. Federal Environmental Agency, Germany.

Makris, S., et al. (1998). A retrospective analysis of twelve developmental neurotoxicity studies submitted to the USEPA Office of Prevention, Pesticides, and Toxic Substances (OPPTS). Available at www.epa.gov/scipoly/sap/1998/index.htm.

NAS (National Academy of Sciences). (1983). Risk Assessment in the Federal Government: Managing the Process. National Academy Press, Washington, DC.

Naumann, B. D. (2001). Studies of categorical data-derived adjustment factors. HERA 7(1), 61-106.

OECD. (1997). Screening information data set (SIDS) manual for the OECD programme on the co-operative investigation of high production volume chemicals, Third revision. OECD Secretariat, July.

Plunkett, L. M. (1999). Do current FIFRA testing guidelines protect infants and children? Lead as a case study. Regul. Toxicol. Pharmacol. 29, 80-87.

Rane, A. (1992). Drug disposition and action in infants and children. In Pediatric Pharmacology: Therapeutic Principles in Practice (S. J. Yaffe and J. V. Aranda, Eds.), pp. 10-21. Saunders, Philadelphia.

Renwick, A. G. (1998). Toxicokinetics in infants and children in relation to the ADI and TDI. Food Addit. Contam. 15, 17-35. 
Renwick, A. G., and Lazarus, N. R. (1998). Human variability and noncancer risk assessment-An analysis of the default uncertainty factor. Regul. Toxicol. Pharmacol. 27(1 Pt. 1), 3-20.

Renwick, A. G., et al. (2000). An analysis of the need for an additional uncertainty factor for infants and children. Regul. Toxicol. Pharmacol. 31, 286-296.

Renwick, A. G., et al. (2001). Pathway-related factors: The potential for human data to improve the scientific basis of risk assessment. HERA 7(1), 165-180.

Rhomberg, L. R. (1997). A survey of the methods for chemical health risk assessment among federal regulatory agencies. HERA 3 , 1029-1196.

Science Advisory Panel (SAP). (1999). Report of the FIFRA Scientific Advisory Panel Meeting, A Retrospective Analysis of Developmental Neurotoxicity Studies. Report No. 99-01B. Arlington, VA.

Sheehan, D. M., and Gaylor, D. W. (1990). Analysis of the adequacy of safety factors. Teratology 41, 590-591.

Silverman, K. C., et al. (1999). Establishing data-derived adjustment factors from published pharmaceutical clinical trial data. HERA $5(5), 1059-1089$.
Skowronski, G. A., and Abdel-Rahman, M. 2001. Relevance of IOX uncertainty factor to the risk assessment of drugs used by children and geriatrics. HERA 7(1), 139-152.

U.S. EPA. (1991). U.S. Environmental Protection Agency. Pesticide Assessment Guidelines, Addendum 10-Neurotoxicity, Washington, DC.

U.S. EPA. (1998). U.S. Environmental Protection Agency. Health Effects Test Guideline OPPTS 870.6300: Developmental Neurotoxicity Study. Office of Prevention, Pesticides and Toxic Substances. EPA 712-C-98-239, Washington, DC.

U.S. EPA. (2002). U.S. Environmental Protection Agency. Determination of the Appropriate FQPA Safety Factor(s) in Tolerance Assessment. Office of Pesticide Programs, Washington, DC.

U.S. EPA. (2001). Integrated Risk Information System (IRIS). United States Environmental Protection Agency. Available online, www.epa.gov/iris.

Weil, C. S. (1972). Statistics vs safety factors and scientific judgment in the evaluation of safety for man. Toxicol. Appl. Pharmacol. 21, 454-463. 